\title{
Coordinated Quality Control in a Two-Stage System *
}

\author{
David D. Yao ${ }^{\dagger}$ \\ Department of Industrial Engineering and Operations Research \\ Columbia University, New York, NY 10027-6699 \\ Shaohui Zheng $\ddagger$ \\ Department of Information and Systems Management \\ Hong Kong University of Science and Technology \\ Clear Water Bay, Kowloon, Hong Kong
}

(July 1997; Revised: June 1998)

\begin{abstract}
We study a quality control problem in a two-stage system. We assume that at each stage units are processed in batches, and the rates are random variables with known distributions. Final products are supplied to customers under warranties or service contracts, with penalty costs associated with defective units. Our focus is on coordinating the inspection procedures at the two stages. Using a stochastic dynamic programming approach, we show that the optimal policy at stage 1 is characterized by a sequence of thresholds, and at stage 2 , by a priority structure, as well as a threshold structure. The key to optimality is a so-called $K$-submodularity property, which is a strengthening of the usual notion of submodularity.

Key words: stochastic dynamic programming, submodularity, sequential threshold policy, quality control.
\end{abstract}

\section{Introduction}

Recently, Chen, Yao and Zheng [6] studied the quality control of a batch of finished products supplied to customers under warranty. The units in the batch are either defective or nondefective, with different lifetime distributions. The defect rate - the proportion of defective units in the batch - is itself a random variable, with a given distribution. Defective units can be identified and repaired through inspection. On the other hand, uninspected units could be defective, and defectives will incur a penalty in the form of warranty cost or after-sales service

\footnotetext{
*An abridge version of this paper appears in the Proceedings of IEEE 36th Conference on Decision and Control, San Diego, CA, 1997.

${ }^{\dagger}$ Research supported in part by NSF Grant DMI-9523029. < yao@columbia.edu >

${ }^{\ddagger}$ Research supported in part by Hong Kong RGC Competitive Earmarked Research Grant HKUST6220/97H. $<$ imzheng@usthk.ust.hk>
} 
cost. The problem is to find an inspection policy for the batch that strikes an optimal balance between the inspection-repair cost and the warranty cost.

A sequential inspection procedure is developed in [6], and proven to be optimal for a very general class of warranty cost functions, and without any distributional restrictions on either the defect rate or the lifetimes of the products. Specifically, the optimal policy is characterized by a sequence of thresholds: $\left\{d_{n}^{*}, n=1, \ldots, N\right\}$, where $N$ is the size of the batch. Let $D(n)$ be the number of defective units identified when $n$ units have been inspected. The policy works as follows: Inspect the units one at a time; each time compare $D(n)$ against $d_{n}^{*}$, and stop inspection the first time when $D(n)<d_{n}^{*}$ (i.e., ship the whole batch without inspecting the remaining units).

Here we extend the model of [6] to a two-stage serial system: A first stage produces a batch of semi-products, which, after inspection, is sent to a second, final stage. The second stage turns the batch into end-products, and after inspection supplies the batch to customer demand. The focus here is on the coordination of the inspection procedures at the two stages.

In many production systems the inspection can be represented as a two-stage scheme. For instance, in the context of semiconductor wafer fabrication, the first stage corresponds to wafer probe, an important step that involves computer-controlled automatic testing; while the second stage involves a more routine, final inspection; refer to Lee [10]; also Walker [17]. Other related recent studies include: Cassandras [5], on "yield learning," focusing on threshold control policies; and Yao and Zheng [18], on process control using Markov decision programming, and also focusing on the optimality of threshold policies.

We adopt the same setting as in [6], in particular, the finished products will be supplied under warranties or some type of service contracts. The added dimension here is that the two stages are closely coupled. Decisions at the first stage have to take into account the inspection policy of the second stage when the batch is sent downstream. For the second stage, the batch carries different types of defects: those due to either the first stage or the second stage or both, and the overall quality of the batch also depends on the inspection policy of the first stage. For instance, if the first stage inspects more units, then there will be fewer units carrying stage 1 defect in the batch.

In the literature, most studies on quality control in multi-stage systems assume that the defect rate is a known constant. For instance, Lindsay and Bishop [11], assuming a constant defect rate at each stage, and with defective units disposed at a cost, showed that the optimal policy is either $100 \%$ or $0 \%$ inspection at each stage. Eppen and Hurst [7] generalized the model of [11] to allow imperfect inspection. Ballou and Pazer [2] further discussed the impact of inspector fallibility on the optimal policy under imperfect inspection. 
The assumption of a constant defect rate leads to a binomial distribution of the number of defective units in the batch. As the batch size becomes large, the coefficient of variation of the number of defective units can be arbitrarily small, making the model inappropriate in some applications; refer to, e.g., Albin and Friedman [1]. Furthermore, the constant defect rate leads to a static, parametric optimization problem, rather than a dynamic control problem, since there is no update in term of the quality information of the batch as inspection progresses.

Our approach is stochastic dynamic programming (refer to, e.g., Bertsekas [3], and Ross [13]). We focus on the optimality of a class of policies that possess a sequential structure, characterized by a sequence of thresholds. The optimality of threshold policies in the control of discrete-event stochastic systems is the subject of a recent work, Glasserman and Yao [8], where it is shown that submodularity of the cost objective, along with certain combinatorial properties that underlie the structure of the system, generally leads to the optimality of simple, monotone policies that are characterized by thresholds or switching curves. In our problem here, we find it necessary to focus on the so-called $K$-submodularity, first developed in [6], which is a strengthening of the more standard submodularity property. Consequently, the resulting optimal policy has an "optimal stopping" flavor (e.g. Wald [16]).

The rest of the paper is organized as follows. In $\S 2$ we present the two-stage model focusing on the case of an additive penalty cost function (which corresponds to individual warranties those that applied to individual units instead of a whole batch of units). The optimal policies for both stages are developed in the next two sections: $\S 3$ for the first stage and $\S 4$ for the second stage. In particular, we show that the optimal policy at the first stage, under a key Condition 2.2 , has the same form as in the single-stage model of [6]: it is a threshold policy characterized by a sequence of thresholds. For the second stage, the optimal policy is to give priority to those units that have not been inspected by the first stage. Furthermore, it is also characterized by a two-dimensional threshold structure. We consider the special case of constant defect rates at both stages in $\S 5$, and revisit the optimal policy at stage 1 in $\S 6$. In $\S 7$, we extend the results to more general cost functions. Throughout, we use the terms "increasing" and "decreasing" in the non-strict sense, to mean non-decreasing and non-increasing, respectively.

\section{Model Description and Preliminaries}

\subsection{A Two-Stage Quality Control Model}

We study quality control in the following system of two stages in tandem. The first stage feeds batches of semi-products into the second stage, which supplies customer demand, under some type of warranty or service contract. Specifically, a batch of $N$ units is first processed and 
inspected at stage 1 and then passed on to stage 2 for processing and inspection. The defect rate at the two stages are modeled as random variables, $\Theta_{1}$ and $\Theta_{2}$. We focus on the inspection processes at the two stages, ignoring the production aspects, except taking into account the possibility of creating defective units. There are three types of defective units in the system: those that carry either a stage 1 defect or a stage 2 defect and those that carry both. We shall refer to the last type as "1-2 defect". In particular, the batch inspected at stage 1 only carries stage 1 defects, while the batch inspected at stage 2 can carry all three types of defects: in particular, the defective units unidentified at stage 1 can further carry a stage 2 defect as well. Let $Y_{1}, Y_{2}$ and $Y_{12}$ denote the lifetimes of a unit that carries one of the three types of defects; let $X$ denote the lifetime of a good (i.e., non-defective) unit. Assume, for $i=1,2$, $X \geq_{\text {st }} Y_{i} \geq_{\text {st }} Y_{12}$. (Refer to the next subsection for the stochastic ordering $\geq_{\text {st }}$.)

Assume at both stages inspection and repair are perfect, and each identified defective unit is repaired. For each unit inspected by both stages, it has a lifetime $X$. On the other hand, if a unit has not been inspected at either stage, then its lifetime is either $Y_{1}, Y_{2}, Y_{12}$ or $X$, respectively with probabilities $\Theta_{1}\left(1-\Theta_{2}\right), \Theta_{2}\left(1-\Theta_{1}\right), \Theta_{1} \Theta_{2}$, and $\left(1-\Theta_{1}\right)\left(1-\Theta_{2}\right)$. Hence, to model the stage- 1 defect, for instance (and likewise for stage- 2 defect), we can first draw a sample from the distribution of $\Theta_{1}$. Given this sample value, each unit is then defective or not with a probability equal to this sample value. This way, the dependence of all $N$ units in the same batch is quite naturally captured through the random defect rate.

Suppose that the inspection costs are respectively $c_{i 1}$ and $c_{i 2}$ per unit at the two stages, and the repair costs are $c_{r 1}, c_{r 2}$ and $c_{r 12}$ per unit respectively for the three types of defects. Assume $c_{r 12} \geq c_{r 1}+c_{r 2}$.

Suppose the warranty cost associated with each unit is either $C\left(Y_{1}\right), C\left(Y_{2}\right), C\left(Y_{12}\right)$, or $C(X)$, depending on whether or not the unit is defective, and if it is, what type of defect it carries. (More general cost functions are also possible; refer to $\S 7$.) Naturally, assume $C(\cdot)$ is a decreasing function (of the unit's lifetime). In addition, we also assume the following relations:

$$
\mathrm{E} C\left(Y_{1}\right) \geq \mathrm{E} C(X)+c_{r 1}, \quad \mathrm{E} C\left(Y_{2}\right) \geq \mathrm{E} C(X)+c_{r 2}
$$

and

$$
\mathrm{E} C\left(Y_{12}\right) \geq \max \left\{\mathrm{E} C\left(Y_{1}\right)+c_{r 12}-c_{r 1}, \mathrm{E} C\left(Y_{2}\right)+c_{r 12}-c_{r 2}\right\}
$$

The above simply give adequate incentive for repairing defective units. Note in particular that the inequalities in (1) and (2) imply

$$
\mathrm{E} C\left(Y_{12}\right) \geq \mathrm{E} C(X)+c_{r 12} .
$$


Furthermore, we assume that the following relation holds:

$$
\mathrm{E} C\left(Y_{12}\right)+\mathrm{E} C(X)-\mathrm{E} C\left(Y_{1}\right)-\mathrm{E} C\left(Y_{2}\right) \geq 0 .
$$

Note that the above can be rewritten as:

$$
\mathrm{E} C\left(Y_{12}\right)-\mathrm{E} C(X) \geq\left[\mathrm{E} C\left(Y_{1}\right)-\mathrm{E} C(X)\right]+\left[\mathrm{E} C\left(Y_{2}\right)-\mathrm{E} C(X)\right]
$$

That is, the cost penalty (i.e., the increment above the usual service cost of a good unit) for a type-12 defect is at least as much as the cost penalty of a type 1 and a type 2 defect combined.

Our problem here is to find inspection policies for both stage 1 and stage 2 so as to minimize the expected total cost (inspection, repair and warranty costs) for each batch of $N$ units, at either stage. We start with some preliminary analysis in the next subsection.

\subsection{Stochastic Order Relations}

We are particularly interested in the optimality of certain sequential inspection policies, at both stages. The main advantage of these policies is they make full use of the information, obtained via inspection, on the quality of the batch in question. Specifically, a sequential policy works as follows: Each time a unit is inspected, the posterior (conditional) distribution of the defect rate is updated, and a decision whether or not to continue inspection is made accordingly. Since this applies to both stages, here we omit the indices (subscripts) for the two stages. Let $D(n)$ be the number of defectives identified from inspecting $n$ items. Then, $[\Theta \mid D(n)=d]$, for some integer $d$ : $0 \leq d \leq n$, denotes the conditional distribution of $\Theta$, updated after $n$ units have been inspected. We are interested in the monotone behavior of this conditional distribution, with respect to $n$ and $d$.

We need some preliminaries on stochastic order relations. The facts summarized below are available in, e.g., Ross [12], Chapter 8. The likelihood ratio ordering among two random variables $X$ and $Y$, denoted $X \geq_{\operatorname{lr}} Y$, is defined as $f_{X}(x) / f_{Y}(x) \geq f_{X}(y) / f_{Y}(y)$ for all $x \geq y$, where $f_{X}$ and $f_{Y}$ denote the density functions of $X$ and $Y$. (For discrete random variables, the likelihood ratio ordering is similarly defined, with the density functions replaced by probability mass functions. ) It is known that $X \geq_{\operatorname{lr}} Y$ implies $X \geq_{\text {st }} Y$, with the latter denoting the stochastic ordering, defined as $F_{X}(x) \leq F_{Y}(x)$ for all $x$, where $F_{X}$ and $F_{Y}$ denote the distribution functions of $X$ and $Y$. It is also known that $X \geq_{\text {st }} Y$ if and only if $\mathrm{E}[g(X)] \geq \mathrm{E}[g(Y)]$ for any function $g(x)$ that is increasing in $x$.

The lemma below establishes that the likelihood ratio ordering applies to the conditional random variable $[\Theta \mid D(n)=d]$, as $n$ and $d$ varies. This then implies the weaker, stochastic ordering (which, paradoxically, is more cumbersome to prove directly). 
Lemma 2.1 For all $n$ and $d \leq n$, we have

$$
\begin{aligned}
{[\Theta \mid D(n)=d+1] } & \geq \operatorname{lr} \quad[\Theta \mid D(n+1)=d+1] \\
& \geq \operatorname{lr} \quad[\Theta \mid D(n)=d] \\
& \geq \operatorname{lr} \quad[\Theta \mid D(n+1)=d] .
\end{aligned}
$$

In particular, $[\Theta \mid D(n)=d]$ is increasing in $d$ and decreasing in $n$, both in the sense of the likelihood ratio ordering (and hence, also in the sense of stochastic ordering).

Proof. To be specific, suppose $\Theta$ has a density function $f_{\Theta}(x)$. Then, the density function of $[\Theta \mid D(n)=d]$, denoted $f_{(n, d)}(x)$ can be derived as:

$$
f_{(n, d)}(x)=\frac{x^{d}(1-x)^{n-d} f_{\Theta}(x)}{\mathrm{E}\left[\Theta^{d}(1-\Theta)^{n-d}\right]} .
$$

We first prove the third inequality. Since

$$
\frac{f_{(n, d)}(x)}{f_{(n+1, d)}(x)}=\frac{1}{1-x} \cdot \frac{\mathrm{E}\left[\Theta^{d}(1-\Theta)^{n+1-d}\right]}{\mathrm{E}\left[\Theta^{d}(1-\Theta)^{n-d}\right]}
$$

is increasing in $x$, the desired likelihood ratio ordering follows. For the second inequality, notice that

$$
\frac{f_{(n+1, d+1)}(x)}{f_{(n, d)}(x)}=x \cdot \frac{\mathrm{E}\left[\Theta^{d}(1-\Theta)^{n-d}\right]}{\mathrm{E}\left[\Theta^{d+1}(1-\Theta)^{n-d}\right]}
$$

is also increasing in $x$. The first inequality is the same as the third one.

\subsection{Convexity and $K$-Submodularity}

Consider the inspection of a batch of $N$ units at stage 1, the first stage. Unlike the single-stage model in [6], here the decisions at stage 1 must take into account the costs that will be incurred downstream at stage 2 , and is more complicated. In fact, the objective function that stage 1 is to optimize will include the cost to be incurred at stage 2 . So in order to formulate the problem at stage 1 , we first need to derive expressions for the cost to be incurred at stage 2 . Let $\phi\left(n_{1}, \theta_{1}\right)$ denote the minimum expected cost to be incurred at stage 2 (including inspection, repair and warranty costs), given $n_{1}$ units have been inspected at stage 1 , and given $\Theta_{1}=\theta_{1}$. Note that here the $\phi$ function, by definition, corresponds to the optimal policy at stage 2 .

Below we list two properties of the $\phi$ function that will result in an optimal threshold policy at stage 1. (Note, however, that these two properties only constitute a sufficient condition for the optimality of a threshold policy; and, in general, they need not be satisfied. See more discussions in $\S 6$, where we show that these properties are satisfied when stage 2 defect is treated as a constant.) 
Condition 2.2 (i) The function $\phi\left(n_{1}, \theta_{1}\right)$ is convex in $n_{1}$. That is, for any given $\theta_{1}$ and any integer $n_{1} \geq 0$,

$$
\phi\left(n_{1}, \theta_{1}\right)-\phi\left(n_{1}+1, \theta_{1}\right) \geq \phi\left(n_{1}+1, \theta_{1}\right)-\phi\left(n_{1}+2, \theta_{1}\right) .
$$

(ii) The function $\phi\left(n_{1}, \theta_{1}\right)$ is $K$-submodular in $\left(n_{1}, \theta_{1}\right)$ with $K=c_{r 1}$. That is, for $\theta_{1}^{\prime} \geq \theta_{1}$,

$$
\left[\phi\left(n_{1}+1, \theta_{1}\right)+\phi\left(n_{1}, \theta_{1}^{\prime}\right)\right]-\left[\phi\left(n_{1}, \theta_{1}\right)+\phi\left(n_{1}+1, \theta_{1}^{\prime}\right)\right] \geq c_{r 1}\left(\theta_{1}^{\prime}-\theta_{1}\right) .
$$

The notion of $K$-submodularity was introduced in [6]. To motivate, recall the more standard submodularity property: A bivariate function, $g(x, y)$, is submodular if for all $x_{1} \geq x_{2}$ and $y_{1} \geq y_{2}$, we have

$$
\left[g\left(x_{1}, y_{2}\right)+g\left(x_{2}, y_{1}\right)\right]-\left[g\left(x_{1}, y_{1}\right)+g\left(x_{2}, y_{2}\right)\right] \geq 0 .
$$

When $g$ is twice differentiable, the above is equivalent to $\partial^{2} g(x, y) / \partial x \partial y \leq 0$ - often a convenient approach to verify submodularity. A submodular function is known to have the following monotone property (e.g., Topkis [15]): suppose $x^{*}(y)$ is the optimal solution to the minimization problem, $\min _{x} g(x, y)$, for a given $y$; then $x^{*}(y)$ is increasing in $y$.

$K$-submodularity is a strengthening of submodularity: A bivariate function, $g(x, y)$, is $K$ submodular, if for some $K \geq 0$, we have,

$$
\left[g\left(x_{1}, y_{2}\right)+g\left(x_{2}, y_{1}\right)\right]-\left[g\left(x_{1}, y_{1}\right)+g\left(x_{2}, y_{2}\right)\right] \geq K\left(x_{1}-x_{2}\right)\left(y_{1}-y_{2}\right),
$$

for all $x_{1} \geq x_{2}$ and $y_{1} \geq y_{2}$. Obviously, $K$-submodularity specializes to submodularity with $K=0$.

From the above definition, we observe the following geometric property of a $K$-submodular function: Consider its values on the four corner points, $\left(x_{1}, y_{1}\right),\left(x_{1}, y_{2}\right),\left(x_{2}, y_{1}\right)$ and $\left(x_{2}, y_{2}\right)$, of a rectangle on the $x-y$ plane; the sum on the southwest-northeast diagonal is greater than the sum on the other diagonal by at least $K$ times the area of the rectangle.

Note that the function $g(x, y)$ is $K$-submodular if and only if $\tilde{g}(x, y)=g(x, y)-K x y$ is a submodular function. In other words, any $K$-submodular function can always be expressed as the sum of a submodular function and $K x y$. Since the sum of two submodular functions are submodular, the sum of a submodular and a $K$-submodular function is $K$-submodular.

Also note that another related property is subadditivity. Generally speaking, in the control of many discrete-event stochastic systems, subadditivity is useful in establishing stability results (e.g., [9]), where as submodularity is key to the optimality of control policies that have a monotone structure $([8])$. 


\section{$3 \quad$ Analysis of Stage 1}

Here we identify the optimal policy at stage 1 under Condition 2.2. Specifically, we assume that given $n$ units have been inspected at stage 1 , and given the defect rate $\Theta_{1}=\theta_{1}$, the expected cost to be incurred at stage 2 (following an optimal policy there), denoted $\phi\left(n_{1}, \theta_{1}\right)$, satisfies the convexity and submodularity assumed in Condition 2.2 .

With these properties, the optimal policy at stage 1 is characterized by a sequence of thresholds, similar to the optimal policy in the single-stage model of [6]; see Theorem 3.3 below. The structure of this optimal policy is a consequence of the properties listed in Lemma 3.1 and Theorem 3.2.

Since we are dealing with the first stage exclusively in this section, we shall omit the subscript 1 , with the exception of certain primitive data such as $\Theta_{1}, c_{i 1}, c_{r 1}$, etc. We say stage 1 is in state $(n, d)$, for $0 \leq n \leq N$ and $d \leq n$, if $n$ units have been inspected, of which $d$ are found defective. Denote $V_{n}(d)$ as the optimal (expected) future cost, starting from $(n, d)$, which includes the possible inspection and rework costs in both stages, and the warranty cost for the whole batch. Let $\Phi_{n}(d)$ and $\Psi_{n}(d)$ denote the expected future costs, starting from the state $(n, d)$, respectively for the two actions, to stop and to continue inspection. Let $D(n)$ denote the number of defective units found in inspecting $n$ units (at stage 1). Then,

$$
\begin{gathered}
\Phi_{n}(d)=\mathrm{E}\left[\phi\left(n, \Theta_{1}\right) \mid D(n)=d\right], \\
\Psi_{n}(d)=c_{i 1}+c_{r 1} \mathrm{E}\left[\Theta_{1} \mid D(n)=d\right]+\mathrm{E}\left[V_{n+1}(D(n+1)) \mid D(n)=d\right] ;
\end{gathered}
$$

whereas,

$$
V_{n}(d)=\min \left\{\Phi_{n}(d), \Psi_{n}(d)\right\}, \quad n<N ; \quad \text { and } \quad V_{N}(d)=\Phi_{N}(d) .
$$

(The last equation above is due to the fact that when all $N$ units are inspected, the only option is to stop inspection.)

From standard results in stochastic dynamic programming (e.g., $[3,13])$, we know that in each state $(n, d)$, it is optimal to continue inspection if and only if $\Psi_{n}(d) \leq \Phi_{n}(d)$. Our problem here is to find a policy that minimizes $V_{0}(0)$.

Lemma 3.1 Under Condition 2.2,

$$
\mathrm{E}\left[\Phi_{n+1}(D(n+1)) \mid D(n)=d\right]-\Phi_{n}(d)+c_{r 1} \mathrm{E}\left[\Theta_{1} \mid D(n)=d\right]
$$

is decreasing in $d$ and increasing in $n$. 
Proof. From (5), decreasing in $d$ in (8) means that for any $d \leq d^{\prime}$, the following holds

$$
\begin{aligned}
\mathrm{E}\left[\phi\left(n+1, \Theta_{1}\right)-\phi\left(n, \Theta_{1}\right)+c_{r 1} \Theta_{1} \mid D(n)=d\right] \\
\geq \mathrm{E}\left[\phi\left(n+1, \Theta_{1}\right)-\phi\left(n, \Theta_{1}\right)+c_{r 1} \Theta_{1} \mid D(n)=d^{\prime}\right] .
\end{aligned}
$$

Let

$$
g(\theta):=\phi(n+1, \theta)-\phi(n, \theta)+c_{r 1} \theta .
$$

From Condition 2.2(ii), we know that $g(\theta)$ is decreasing in $\theta$. On the other hand, from Lemma 2.1 , we know

$$
\left[\Theta_{1} \mid D(n)=d\right] \leq_{\mathrm{st}}\left[\Theta_{1} \mid D(n)=d^{\prime}\right]
$$

for any $d \leq d^{\prime}$. Hence,

$$
\mathrm{E}\left[g\left(\Theta_{1}\right) \mid D(n)=d\right] \geq_{\text {st }} \mathrm{E}\left[g\left(\Theta_{1}\right) \mid D(n)=d^{\prime}\right]
$$

which yields the desired relation in (9).

Next, $\left[\Theta_{1} \mid D(n-1)=d\right] \geq_{\text {st }}\left[\Theta_{1} \mid D(n)=d\right]$, following Lemma 2.1. Hence, we have

$$
\begin{aligned}
& \mathrm{E}\left[\phi\left(n+1, \Theta_{1}\right)-\phi\left(n, \Theta_{1}\right)+c_{r 1} \Theta_{1} \mid D(n)=d\right] \\
\geq & \mathrm{E}\left[\phi\left(n+1, \Theta_{1}\right)-\phi\left(n, \Theta_{1}\right)+c_{r 1} \Theta_{1} \mid D(n-1)=d\right] \\
\geq & \mathrm{E}\left[\phi\left(n, \Theta_{1}\right)-\phi\left(n-1, \Theta_{1}\right)+c_{r 1} \Theta_{1} \mid D(n-1)=d\right],
\end{aligned}
$$

where the first inequality follows from the decreasingness of $g(\theta)$, and the second inequality follows from Condition 2.2 (i). Thus, we have shown that (8) is increasing in $n$.

Theorem 3.2 Under Condition 2.2, for stage 1,

- if it is optimal to continue inspection in state $(n, d)$, then it is also optimal to continue inspection in state $(n, d+1)$;

- if it is optimal to stop inspection in $(n, d)$, then it is also optimal to stop inspection in $(n+1, d)$.

In particular, $\Psi_{n}(d)-\Phi_{n}(d)$ is decreasing in $d$ (for each given $n$ ), and increasing in $n$ (for each given $d$ ).

Proof. Note that it is optimal to continue inspection in any state $(n, d)$ if and only if $\Psi_{n}(d) \leq$ $\Phi_{n}(d)$. Hence, to prove the first statement amounts to proving the following: given $n$, for each $d<n$,

$$
\Psi_{n}(d) \leq \Phi_{n}(d) \quad \Rightarrow \quad \Psi_{n}(d+1) \leq \Phi_{n}(d+1)
$$


Below, we prove a stronger result: that $\Psi_{n}(d)-\Phi_{n}(d)$ is decreasing in $d$, for each given $n$. We argue via induction on $n$.

When $n=N-1$, we have

$$
\mathrm{E}\left[V_{n+1}(D(n+1)) \mid D(n)=d\right]=\mathrm{E}\left[\phi\left(n+1, \Theta_{1}\right) \mid D(n)=d\right] .
$$

Hence, from (5) and (6), we have

$$
\Psi_{n}(d)-\Phi_{n}(d)=c_{i 1}+\mathrm{E}\left[c_{r 1} \Theta_{1}+\phi\left(n+1, \Theta_{1}\right)-\phi\left(n, \Theta_{1}\right) \mid D(n)=d\right],
$$

which is decreasing in $d$, following Lemma 3.1. Since $V_{n}(d)=\min \left\{\Phi_{n}(d), \Psi_{n}(d)\right\}$, we have

$$
V_{n}(d)-\Phi_{n}(d)=\min \left\{0, \Psi_{n}(d)-\Phi_{n}(d)\right\}
$$

Hence, $V_{n}(d)-\Phi_{n}(d)$ is also decreasing in $d$.

Next, consider $n<N-1$. As induction hypothesis, assume that $\Psi_{n+1}(d)-\Phi_{n+1}(d)$, and hence $V_{n+1}(d)-\Phi_{n+1}(d)$, is decreasing in $d$. Note that

$$
\begin{aligned}
& \Psi_{n}(d)-\Phi_{n}(d) \\
= & c_{i 1}+\mathrm{E}\left[c_{r 1} \Theta_{1}+V_{n+1}(D(n+1))-\phi\left(n, \Theta_{1}\right) \mid D(n)=d\right] \\
= & c_{i 1}+\mathrm{E}\left[c_{r 1} \Theta_{1}+\phi\left(n+1, \Theta_{1}\right)-\phi\left(n, \Theta_{1}\right) \mid D(n)=d\right] \\
& +\mathrm{E}\left[V_{n+1}(D(n+1))-\phi\left(n+1, \Theta_{1}\right) \mid D(n)=d\right] .
\end{aligned}
$$

The first expectation following the second equation above is decreasing in $d$, following Lemma 3.1. Hence it remains to show that the second expectation is also decreasing in $d$. Rewrite it as follows:

$$
\mathrm{E}\left[V_{n+1}\left(d+I_{n}(d)\right)-\Phi_{n+1}\left(d+I_{n}(d)\right)\right]
$$

where $I_{n}(d)$ is a binary random variable that equals 1 with probability $\mathrm{E}\left[\Theta_{1} \mid D(n)=d\right]$. Since $I_{n}(d)$ is stochastically increasing in $d$, following Lemma 2.1, the expectation in (11) is decreasing in $d$, following the induction hypothesis that $V_{n+1}(d)-\Phi_{n+1}(d)$ is decreasing in $d$.

To prove the second statement in the Theorem, it suffices to show that $\Psi_{n}(d)-\Phi_{n}(d)$ is increasing in $n$, given $d$. Make use of (10). The first expectation is increasing in $n$, following Lemma 3.1. To argue that the expectation in (11) is also increasing in $n$, note that $I_{n}(d)$ is stochastically decreasing in $n$, following Lemma 2.1 , and that $V_{n+1}(d)-\Phi_{n+1}(d)$ is decreasing in $d$.

Theorem 3.3 For $n=1, \ldots, N$, define $d_{n}^{*}:=\min \left\{d \leq n: \Psi_{n}(d) \leq \Phi_{n}(d)\right\}$. Then, under Condition 2.2, 
(i) $d_{n}^{*}$ is increasing in $n$;

(ii) the optimal policy for inspection at stage 1 is to inspect the units one unit at a time, and stop as soon as a state $(n, d)$ is reached with $d<d_{n}^{*}$.

Proof. Part (i) follows directly from the fact that $\Psi_{n}(d)-\Phi_{n}(d)$ is increasing in $n$ and decreasing in $d$ (Theorem 3.2). For part (ii), observe that from the definition of $d_{n}^{*}, d<d_{n}^{*}$ implies $\Psi_{n}(d)>\Phi_{n}(d)$. Hence, it is optimal to stop inspection in $(n, d)$.

\section{Optimal Policy at Stage 2}

The inspection process at stage 2 is considerably different from stage 1 . The quality of the batch depends on the inspection at stage 1 , and there are several different types of defects. Suppose that the units inspected at stage 1 are marked and identified with a set $\mathbf{A}$. The first problem we face is how to deal with these units: should they be given higher or lower priority than the units in the complement set $\overline{\mathbf{A}}$, or should the two sets not be distinguished by priorities at all?

This section is divided into two parts. In the first part, we address the issue of priority, and in the second part, we present the complete optimal policy for stage 2 .

\subsection{Priority Structure}

To start with, consider the decision: whether or not to inspect a particular unit at stage 2, given the defect rates at both stages are known constants: $\Theta_{1}=\theta_{1}$ and $\Theta_{2}=\theta_{2}$. Let $R_{1}\left(\theta_{1}, \theta_{2}\right)$ and $R_{0}\left(\theta_{1}, \theta_{2}\right)$ denote the expected costs - only for the unit in question - corresponding to the two actions (at stage 2), to inspect and not to inspect, respectively. Then, clearly,

$$
\begin{aligned}
R_{1}\left(\theta_{1}, \theta_{2}\right)= & c_{i 2}+c_{r 1} \theta_{1}\left(1-\theta_{2}\right)+c_{r 2}\left(1-\theta_{1}\right) \theta_{2}+c_{r 12} \theta_{1} \theta_{2}+\mathrm{E} C(X) \\
= & c_{i 2}+c_{r 1} \theta_{1}+c_{r 2} \theta_{2}+\left(c_{r 12}-c_{r 1}-c_{r 2}\right) \theta_{1} \theta_{2}+\mathrm{E} C(X), \\
R_{0}\left(\theta_{1}, \theta_{2}\right)= & \left(1-\theta_{1}\right)\left(1-\theta_{2}\right) \mathrm{E} C(X)+\theta_{1}\left(1-\theta_{2}\right) \mathrm{E} C\left(Y_{1}\right) \\
& +\theta_{2}\left(1-\theta_{1}\right) \mathrm{E} C\left(Y_{2}\right)+\theta_{1} \theta_{2} \mathrm{E} C\left(Y_{12}\right) .
\end{aligned}
$$

From the above two expressions, we have

$$
\begin{aligned}
& R_{1}\left(\theta_{1}, \theta_{2}\right)-R_{0}\left(\theta_{1}, \theta_{2}\right) \\
=c_{i 2} & +\left[\mathrm{E} C(X)-\mathrm{E} C\left(Y_{1}\right)+c_{r 1}\right] \theta_{1}\left(1-\theta_{2}\right) \\
& +\left[\mathrm{E} C(X)-\mathrm{E} C\left(Y_{2}\right)+c_{r 2}\right] \theta_{2}+\left[\mathrm{E} C\left(Y_{2}\right)-\mathrm{E} C\left(Y_{12}\right)+c_{r 12}-c_{r 2}\right] \theta_{1} \theta_{2} \\
=c_{i 2} & +\left[\mathrm{E} C(X)-\mathrm{E} C\left(Y_{1}\right)+c_{r 1}\right] \theta_{1} \\
& +\left[\mathrm{E} C(X)-\mathrm{E} C\left(Y_{2}\right)+c_{r 2}\right]\left(1-\theta_{1}\right) \theta_{2}+\left[\mathrm{E} C\left(Y_{1}\right)-\mathrm{E} C\left(Y_{12}\right)+c_{r 12}-c_{r 1}\right] \theta_{1} \theta_{2} .
\end{aligned}
$$


From the two equalities above, we observe that the expressions are decreasing in $\theta_{1}$ and in $\theta_{2}$, since the relevant coefficients of $\theta_{1}$ and $\theta_{2}$ are all non-positive, taking into account (1) and (2). Furthermore, from (13), we have

$$
\begin{aligned}
& R_{0}\left(\theta_{1}, \theta_{2}\right)-R_{0}\left(0, \theta_{2}\right) \\
= & \theta_{1}\left[\mathrm{E} C\left(Y_{1}\right)-\mathrm{E} C(X)\right]+\theta_{1} \theta_{2}\left[\mathrm{E} C\left(Y_{12}\right)+\mathrm{E} C(X)-\mathrm{E} C\left(Y_{1}\right)-\mathrm{E} C\left(Y_{2}\right)\right] .
\end{aligned}
$$

Hence, the above is increasing in $\theta_{2}$, following (1) and (4). To summarize, we have

Lemma 4.1 (i) $R_{1}\left(\theta_{1}, \theta_{2}\right)-R_{0}\left(\theta_{1}, \theta_{2}\right)$ is decreasing in $\theta_{1}$ and $\theta_{2}$.

(ii) $R_{0}\left(\theta_{1}, \theta_{2}\right)-R_{0}\left(0, \theta_{2}\right)$ is increasing in $\theta_{2}$.

The following theorem assures that we only need to consider policies (at stage 2) that give priority to units in the set $\overline{\mathbf{A}}$.

Theorem 4.2 For stage 2, there exists an optimal policy that does not inspect any unit already inspected at stage 1 (i.e., those in set $\mathbf{A}$ ) until all the other units (in $\overline{\mathbf{A}}$ ) are inspected.

To better understand the above theorem as well as to prepare for its proof, we pursue the statement of the theorem a little further so as to reduce it to an inequality [in (14)]. Suppose $|\mathbf{A}|=n_{1}$ units in the batch have already been inspected at stage 1 . In addition, stage 2 has inspected $n_{2}$ units from $\mathbf{A}$ and $\bar{n}_{2}$ units from $\overline{\mathbf{A}}$. It is important to keep in mind that the units inspected at stage 2 from $\mathbf{A}$ can only carry a stage 2 defect, since any possible stage 1 defect have already been corrected at stage 1 . On the other hand, the units inspected at stage 2 from $\overline{\mathbf{A}}$ can still carry all three types of defects.

We shall use $D_{1}(n)$ and $D_{2}(n)$, the number of defective units identified through inspecting $n$ units, to update our knowledge on $\Theta_{1}$ and $\Theta_{2}$. Note that $D_{1}$ includes the units that carry a stage 1 defect identified at both stage 1 and stage 2 , and the units that carry a 1-2 defect (identified at stage 2). Similarly, $D_{2}$ includes the units that carry a stage 2 defect or a 1-2 defect (both identified at stage 2). Note that each unit that carries a 1-2 defect is counted in both $D_{1}$ and $D_{2}$. Thus, $D_{1}\left(n_{1}+\bar{n}_{2}\right)=d_{1}$ means that among a total of $n_{1}+\bar{n}_{2}$ units inspected at both stage 1 and stage $2, d_{1}$ units are found to carry a stage 1 defect; $D_{2}\left(n_{2}+\bar{n}_{2}\right)=d_{2}$ means that among a total of $n_{2}+\bar{n}_{2}$ units inspected at stage $2, d_{2}$ units are found to carry a stage 2 defect. Below, $\left(n_{2}, \bar{n}_{2}, d_{1}, d_{2}\right)$ denotes the state of interest. (Note, since $n_{1}=|\mathbf{A}|$ is given and remains unchanged, we do not include it in the state description.) 
Let $r\left(\theta_{1}, \theta_{2}\right)$ denote the inspection and possible repair cost when an item is inspected (at stage 2 ), given the defect rates at the two stages are $\theta_{1}$ and $\theta_{2}$, respectively. Then,

$$
\begin{aligned}
r\left(\theta_{1}, \theta_{2}\right) & =c_{i 2}+c_{r 1} \theta_{1}\left(1-\theta_{2}\right)+c_{r 2}\left(1-\theta_{1}\right) \theta_{2}+c_{r 12} \theta_{1} \theta_{2} \\
& =R_{1}\left(\theta_{1}, \theta_{2}\right)-\mathrm{E} C(X) .
\end{aligned}
$$

Starting from the state $\left(n_{2}, \bar{n}_{2}, d_{1}, d_{2}\right)$, let $V\left(n_{2}, \bar{n}_{2}, d_{1}, d_{2}\right)$ denote the optimal expected future cost, including inspection, repair and warranty costs. Suppose the decision in state $\left(n_{2}, \bar{n}_{2}, d_{1}, d_{2}\right)$ is to continue inspection. There are two alternatives in selecting the next unit for inspection: from $\overline{\mathbf{A}}$ or from $\mathbf{A}$, with expected future costs respectively as follows:

$$
\begin{aligned}
& \mathrm{E}\left[r\left(\Theta_{1}, \Theta_{2}\right) \mid D_{1}\left(n_{1}+\bar{n}_{2}\right)=d_{1}, D_{2}\left(n_{2}+\bar{n}_{2}\right)=d_{2}\right] \\
& \quad+\mathrm{E}\left[V\left(n_{2}, \bar{n}_{2}+1, d_{1}+I_{1}, d_{2}+I_{2}\right)\right]
\end{aligned}
$$

and

$$
\mathrm{E}\left[r\left(0, \Theta_{2}\right) \mid D_{2}\left(n_{2}+\bar{n}_{2}\right)=d_{2}\right]+\mathrm{E}\left[V\left(n_{2}+1, \bar{n}_{2}, d_{1}, d_{2}+I_{2}^{\prime}\right)\right],
$$

where $I_{1}$ is a binary random variable that equals 1 with probability $\mathrm{E}\left[\Theta_{1} \mid D_{1}\left(n_{1}+\bar{n}_{2}\right)=d_{1}\right]$; similarly, $I_{2}$ and $I_{2}^{\prime}$ are independent samples of another binary random variable that equals 1 with probability $\mathrm{E}\left[\Theta_{2} \mid D_{2}\left(n_{2}+\bar{n}_{2}\right)=d_{2}\right]$. Note that both $\Theta_{2}$ and $I_{2}^{\prime}$ are independent of the event $D_{1}\left(n_{1}+\bar{n}_{2}\right)=d_{1}$.

Therefore, in order to prove Theorem 4.2, it suffices to show that the cost for inspecting a unit from $\overline{\mathbf{A}}$ is smaller, i.e.,

$$
\begin{array}{ll} 
& \mathrm{E}\left[V\left(n_{2}+1, \bar{n}_{2}, d_{1}, d_{2}+I_{2}^{\prime}\right)\right]-\mathrm{E}\left[V\left(n_{2}, \bar{n}_{2}+1, d_{1}+I_{1}, d_{2}+I_{2}\right)\right] \\
\geq \quad & \mathrm{E}\left[r\left(\Theta_{1}, \Theta_{2}\right) \mid D_{1}\left(n_{1}+\bar{n}_{2}\right)=d_{1}, D_{2}\left(n_{2}+\bar{n}_{2}\right)=d_{2}\right] \\
& -\mathrm{E}\left[r\left(0, \Theta_{2}\right) \mid D_{2}\left(n_{2}+\bar{n}_{2}\right)=d_{2}\right] .
\end{array}
$$

We establish the above inequality via induction. We need the following lemma, which is elementary and can be directly verified.

Lemma 4.3 Below, $A, B, a$ and $b$ are all real values.

(i) Suppose $A \geq B \Rightarrow a \geq b$, and $B-b \geq A-a$. Then, $\min \{A, B\}-\min \{a, b\} \geq A-a$.

(ii) Suppose $A-a \geq \Delta$ and $B-b \geq \Delta$ for some $\Delta$. Then, $\min \{A, B\}-\min \{a, b\} \geq \Delta$.

Proof of Theorem 4.2 via (14). To lighten notation, we rewrite (14) as follows:

$$
\begin{aligned}
& \mathrm{E}\left[V\left(1,0,0, I_{2}^{\prime}\right)\right]-\mathrm{E}\left[V\left(0,1, I_{1}, I_{2}\right)\right] \\
\geq & \mathrm{E}\left[r\left(\Theta_{1}, \Theta_{2}\right) \mid D_{1}(0)=d_{1}, D_{2}(0)=d_{2}\right]-\mathrm{E}\left[r\left(0, \Theta_{2}\right) \mid D_{2}(0)=d_{2}\right] .
\end{aligned}
$$


That is, we effectively treat $\left(n_{2}, \bar{n}_{2}, d_{1}, d_{2}\right)$ as the starting point in calculating the future costs.

In what follows, we prove a stronger, sample-path version of the above:

$$
\begin{array}{ll} 
& V\left(1,0,0, I_{2}^{\prime}\right)-V\left(0,1, I_{1}, I_{2}\right) \\
\geq \quad & \mathrm{E}\left[r\left(\Theta_{1}, \Theta_{2}\right) \mid D_{1}(0)=d_{1}, D_{2}(1)=d_{2}+I_{2}^{\prime}\right] \\
& -\mathrm{E}\left[r\left(0, \Theta_{2}\right) \mid D_{2}(1)=d_{2}+I_{2}\right] .
\end{array}
$$

Taking expectations on both sides of (16) recovers (15).

We prove (16) by induction. Since there are uninspected units in both $\mathbf{A}$ and $\overline{\mathbf{A}}$, we have $n_{2}+\bar{n}_{2} \leq N-2$. Consider the case of $n_{2}+\bar{n}_{2}=N-2$. Since there is only one uninspected unit in $\mathbf{A}$ and another in $\overline{\mathbf{A}}$, we have

$$
\begin{array}{r}
V\left(0,1, I_{1}, I_{2}\right)=\min \left\{\mathrm{E}\left[R_{1}\left(0, \Theta_{2}\right) \mid D_{2}(1)=d_{2}+I_{2}\right]+(N-1) \mathrm{E} C(X),\right. \\
\left.\mathrm{E}\left[R_{0}\left(0, \Theta_{2}\right) \mid D_{2}(1)=d_{2}+I_{2}\right]+(N-1) \mathrm{E} C(X)\right\},
\end{array}
$$

and

$$
\begin{array}{r}
V\left(1,0,0, I_{2}^{\prime}\right)=\min \left\{\mathrm{E}\left[R_{1}\left(\Theta_{1}, \Theta_{2}\right) \mid D_{1}(0)=d_{1}, D_{2}(1)=d_{2}+I_{2}^{\prime}\right]+(N-1) \mathrm{E} C(X),\right. \\
\left.\mathrm{E}\left[R_{0}\left(\Theta_{1}, \Theta_{2}\right) \mid D_{1}(0)=d_{1}, D_{2}(1)=d_{2}+I_{2}^{\prime}\right]+(N-1) \mathrm{E} C(X)\right\} .
\end{array}
$$

For convenience, write the two expressions above as

$$
V\left(1,0,0, I_{2}^{\prime}\right)=\min \{A, B\} \quad \text { and } \quad V\left(0,1, I_{1}, I_{2}\right)=\min \{a, b\}
$$

We can then invoke Lemma 4.3 (i). The two required conditions, $A \geq B \Rightarrow a \geq b$ and $B-b \geq A-a$, can be verified as following from Lemma 4.1. Hence, (16) holds, since its right hand side is $A-a$, taking into account the following:

$$
\left[R_{1}\left(\theta_{1}, \theta_{2}\right)+(N-1) \mathrm{E} C(X)\right]-\left[R_{1}\left(0, \theta_{2}\right)+(N-1) \mathrm{E} C(X)\right]=r\left(\theta_{1}, \theta_{2}\right)-r\left(0, \theta_{2}\right) .
$$

As induction hypothesis, suppose (16) holds for $n_{2}+\bar{n}_{2} \geq n+1$. We now show it also holds for $n_{2}+\bar{n}_{2}=n$. Denote as in $\S 3$ the future costs corresponding to continuing and stopping inspection, respectively, as $\Psi$ and $\Phi$. (As it will become evident below, all we need is some differences that involve $\Psi$ and $\Phi$, but not $\Psi$ and $\Phi$ themselves.) We can now express the left hand side of (16) as follows:

$$
\begin{aligned}
& V\left(1,0,0, I_{2}^{\prime}\right)-V\left(0,1, I_{1}, I_{2}\right) \\
= & \min \left\{\Psi\left(1,0,0, I_{2}^{\prime}\right), \Phi\left(1,0,0, I_{2}^{\prime}\right)\right\}-\min \left\{\Psi\left(0,1, I_{1}, I_{2}\right), \Phi\left(0,1, I_{1}, I_{2}\right)\right\} \\
:= & \min \{A, B\}-\min \{a, b\} .
\end{aligned}
$$


Then, we have

$$
\begin{aligned}
& B-b:=\Phi\left(1,0,0, I_{2}^{\prime}\right)-\Phi\left(0,1, I_{1}, I_{2}\right) \\
= & \mathrm{E}\left[R_{0}\left(\Theta_{1}, \Theta_{2}\right) \mid D_{1}(0)=d_{1}, D_{2}(1)=d_{2}+I_{2}^{\prime}\right]-\mathrm{E}\left[R_{0}\left(0, \Theta_{2}\right) \mid D_{2}(1)=d_{2}+I_{2}\right] \\
\geq & \mathrm{E}\left[R_{1}\left(\Theta_{1}, \Theta_{2}\right) \mid D_{1}(0)=d_{1}, D_{2}(1)=d_{2}+I_{2}^{\prime}\right]-\mathrm{E}\left[R_{1}\left(0, \Theta_{2}\right) \mid D_{2}(1)=d_{2}+I_{2}\right] \\
= & \mathrm{E}\left[r\left(\Theta_{1}, \Theta_{2}\right) \mid D_{1}(0)=d_{1}, D_{2}(1)=d_{2}+I_{2}^{\prime}\right]-\mathrm{E}\left[r\left(0, \Theta_{2}\right) \mid D_{2}(1)=d_{2}+I_{2}\right] \\
:= & \Delta,
\end{aligned}
$$

where the inequality follows from Lemma 4.1. In addition,

$$
\begin{aligned}
& A-a:=\Psi\left(1,0,0, I_{2}^{\prime}\right)-\Psi\left(0,1, I_{1}, I_{2}\right) \\
= & \mathrm{E}\left[r\left(\Theta_{1}, \Theta_{2}\right) \mid D_{1}(0)=d_{1}, D_{2}(1)=d_{2}+I_{2}^{\prime}\right]+\mathrm{E}\left[V\left(1,1, I_{1}, I_{2}+I_{2}^{\prime}\right)\right] \\
& \quad-\mathrm{E}\left[r\left(\Theta_{1}, \Theta_{2}\right) \mid D_{1}(1)=d_{1}+I_{1}, D_{2}(1)=d_{2}+I_{2}\right]-\mathrm{E}\left[V\left(0,2, I_{1}, I_{2}+I_{2}^{\prime \prime}\right)\right] \\
\geq & \mathrm{E}\left[r\left(\Theta_{1}, \Theta_{2}\right) \mid D_{1}(0)=d_{1}, D_{2}(1)=d_{2}+I_{2}^{\prime}\right]+\mathrm{E}\left[V\left(1,1, I_{1}, I_{2}+I_{2}^{\prime}\right)\right] \\
& \quad-\mathrm{E}\left[r\left(0, \Theta_{2}\right) \mid D_{2}(1)=d_{2}+I_{2}\right]-\mathrm{E}\left[V\left(1,1, I_{1}, I_{2}+I_{2}^{\prime}\right)\right] \\
= & \mathrm{E}\left[r\left(\Theta_{1}, \Theta_{2}\right) \mid D_{1}(0)=d_{1}, D_{2}(1)=d_{2}+I_{2}^{\prime}\right]-\mathrm{E}\left[r\left(0, \Theta_{2}\right) \mid D_{2}(1)=d_{2}+I_{2}\right] \\
= & \Delta,
\end{aligned}
$$

where the inequality follows from the induction hypothesis. ( $I_{2}^{\prime \prime}$ is another independent sample from the distribution of $I_{2}$ and $I_{2}^{\prime}$.) The desired claim then follows from Lemma 4.3 (ii), noticing that $\Delta$ is exactly the right hand side of (16).

\subsection{Threshold Structure}

From the above discussion, note that in general a full state description for the control at stage 2 is $\left(n_{2}, \bar{n}_{2}, d_{1}, d_{2}\right)$, where $n_{2}$ and $\bar{n}_{2}$ denote the number of units inspected from $\mathbf{A}$ and $\overline{\mathbf{A}}$, and $d_{1}$ and $d_{2}$ denote the number of stage 1 and stage 2 defectives identified respectively. The immediate consequence of Theorem 4.2 is a simplification of the state description. Let $n_{2}$ now denote the total number of units stage 2 has inspected. Then, there is no ambiguity: if $n_{2} \leq N-|\mathbf{A}|$, then all these units are from $\overline{\mathbf{A}}$; otherwise, $N-|\mathbf{A}|$ are from $\overline{\mathbf{A}}$ and the remaining from $\mathbf{A}$. So we remove $\bar{n}_{2}$ from the state description, and let $\left(n_{2}, d_{1}, d_{2}\right)$ denote the new state. We will continue to denote $n_{1}:=|\mathbf{A}|$.

Let $\phi\left(n_{2}, \theta_{1}, \theta_{2}\right)$ denote the expected future cost if stage 2 stops inspection after inspecting a total of $n_{2}$ units, and given $\Theta_{1}=\theta_{1}$ and $\Theta_{2}=\theta_{2}$. Note that $\phi\left(n_{2}, \theta_{1}, \theta_{2}\right)$ is the warranty cost of all the $N$ units, $n_{2}$ of which have been inspected at stage 2, while the other $N-n_{2}$ units have not been inspected. Following Theorem 4.2, there are two cases: for $n_{2} \leq N-n_{1}-1$,

$$
\phi\left(n_{2}, \theta_{1}, \theta_{2}\right)=n_{2} \mathrm{E} C(X)+n_{1} R_{0}\left(0, \theta_{2}\right)+\left(N-n_{1}-n_{2}\right) R_{0}\left(\theta_{1}, \theta_{2}\right) ;
$$


and for $n_{2} \geq N-n_{1}$,

$$
\phi\left(n_{2}, \theta_{1}, \theta_{2}\right)=n_{2} \mathrm{E} C(X)+\left(N-n_{2}\right) R_{0}\left(0, \theta_{2}\right) .
$$

In the first case, all the units inspected are from $\overline{\mathbf{A}}$. The uninspected units then include the $n_{1}$ units in $\mathbf{A}$ and the remaining $N-n_{1}-n_{2}$ units in $\overline{\mathbf{A}}$. In the second case, all the units in $\overline{\mathbf{A}}$ have been inspected. In addition, some units (possibly none) in $\mathbf{A}$ have also been inspected. Hence, the $N-n_{2}$ uninspected units are all in $\mathbf{A}$.

In state $\left(n_{2}, d_{1}, d_{2}\right)$, let $V_{n_{2}}\left(d_{1}, d_{2}\right)$ be the expected total future cost. Let $\Phi_{n_{2}}\left(d_{1}, d_{2}\right)$ and $\Psi_{n_{2}}\left(d_{1}, d_{2}\right)$ be the expected total future costs corresponding to stopping or continuing inspection. We have

$$
V_{n_{2}}\left(d_{1}, d_{2}\right)=\min \left\{\Phi_{n_{2}}\left(d_{1}, d_{2}\right), \Psi_{n_{2}}\left(d_{1}, d_{2}\right)\right\}, \quad n_{2}<N ; \quad V_{N}\left(d_{1}, d_{2}\right)=\Phi_{N}\left(d_{1}, d_{2}\right) ;
$$

where

$$
\Phi_{n_{2}}\left(d_{1}, d_{2}\right)=\mathrm{E}\left[\phi\left(n_{2}, \Theta_{1}, \Theta_{2}\right) \mid D_{1}\left(n_{1}+n_{2}\right)=d_{1}, D_{2}\left(n_{2}\right)=d_{2}\right]
$$

and

$$
\begin{aligned}
\Psi_{n_{2}}\left(d_{1}, d_{2}\right)= & \mathrm{E}\left[r\left(\Theta_{1}, \Theta_{2}\right) \mid D_{1}\left(n_{1}+n_{2}\right)=d_{1}, D_{2}\left(n_{2}\right)=d_{2}\right] \\
& +\mathrm{E}\left[V_{n_{2}+1}\left(d_{1}+I_{1}, d_{2}+I_{2}\right)\right]
\end{aligned}
$$

for $n_{2} \leq N-n_{1}-1$, and

$$
\Psi_{n_{2}}\left(d_{1}, d_{2}\right)=\mathrm{E}\left[r\left(0, \Theta_{2}\right) \mid D_{2}\left(n_{2}\right)=d_{2}\right]+\mathrm{E}\left[V_{n_{2}+1}\left(d_{1}, d_{2}+I_{2}\right)\right]
$$

for $n_{2} \geq N-n_{1}$. Note, in the expressions above,

$$
I_{1}:=\mathbf{1}\left(\mathrm{E}\left[\Theta_{1} \mid D_{1}\left(n_{1}+n_{2}\right)=d_{1}\right]\right), \quad I_{2}:=\mathbf{1}\left(\mathrm{E}\left[\Theta_{2} \mid D_{2}\left(n_{2}\right)=d_{2}\right]\right)
$$

where $\mathbf{1}(p)$ denotes a binary (0-1) random variable that equals one with probability $p$.

The problem here is to find an inspection policy for stage 2 so as to minimize $V_{0}\left(D_{1}\left(n_{1}\right), 0\right)$ for any value of $D_{1}\left(n_{1}\right)$, given $|\mathbf{A}|=n_{1}$.

The lemma below is analogous to Lemma 3.1, and plays a key role in establishing the optimality of the threshold policy for stage 2 .

Lemma 4.4 The following expressions are decreasing in $d_{1}$ and $d_{2}$, and increasing in $n_{2}$ :

$$
\begin{aligned}
& \mathrm{E}\left[\Phi_{n_{2}+1}\left(d_{1}+I_{1}, d_{2}+I_{2}\right)\right]-\Phi_{n_{2}}\left(d_{1}, d_{2}\right) \\
& +\mathrm{E}\left[r\left(\Theta_{1}, \Theta_{2}\right) \mid D_{1}\left(n_{1}+n_{2}\right)=d_{1}, D_{2}\left(n_{2}\right)=d_{2}\right]
\end{aligned}
$$


for $n_{2} \leq N-n_{1}-1$; and

$$
\begin{aligned}
& \mathrm{E}\left[\Phi_{n_{2}+1}\left(d_{1}+I_{1}, d_{2}+I_{2}\right)\right]-\Phi_{n_{2}}\left(d_{1}, d_{2}\right) \\
& +\mathrm{E}\left[r\left(0, \Theta_{2}\right) \mid D_{1}\left(n_{1}+n_{2}\right)=d_{1}, D_{2}\left(n_{2}\right)=d_{2}\right]
\end{aligned}
$$

for $n_{2} \geq N-n_{1}$; where $I_{1}$ and $I_{2}$ follow the definitions in (23).

Proof. The basic idea is similar to the proof of Lemma 3.1. First, for $n_{2} \leq N-n_{1}-1$, let

$$
\begin{aligned}
G_{1}\left(\theta_{1}, \theta_{2}\right) & :=\phi\left(n_{2}+1, \theta_{1}, \theta_{2}\right)-\phi\left(n_{2}, \theta_{1}, \theta_{2}\right)+r\left(\theta_{1}, \theta_{2}\right) \\
& =\mathrm{E} C(X)-R_{0}\left(\theta_{1}, \theta_{2}\right)+r\left(\theta_{1}, \theta_{2}\right) \\
& =R_{1}\left(\theta_{1}, \theta_{2}\right)-R_{0}\left(\theta_{1}, \theta_{2}\right) .
\end{aligned}
$$

Then, $G_{1}\left(\theta_{1}, \theta_{2}\right)$ is decreasing in $\theta_{1}$ and $\theta_{2}$, following Lemma 4.1. On the other hand, from Lemma 2.1, we know that given $n_{1}$ and $n_{2},\left[\Theta_{1} \mid D_{1}\left(n_{1}+n_{2}\right)=d_{1}\right]$ is stochastically increasing in $d_{1}$, and $\left[\Theta_{2} \mid D_{2}\left(n_{2}\right)=d_{2}\right]$ is stochastically increasing in $d_{2}$. Hence,

$$
\mathrm{E}\left[G_{1}\left(\Theta_{1}, \Theta_{2}\right) \mid D_{1}\left(n_{1}+n_{2}\right)=d_{1}, D_{2}\left(n_{2}\right)=d_{2}\right]
$$

is decreasing in $d_{1}$ and $d_{2}$, and so is the expression in (24).

Similarly, for $n_{2} \geq N-n_{1}$, let

$$
\begin{aligned}
G_{0}\left(\theta_{1}, \theta_{2}\right) & :=\phi\left(n_{2}+1, \theta_{1}, \theta_{2}\right)-\phi\left(n_{2}, \theta_{1}, \theta_{2}\right)+r\left(0, \theta_{2}\right) \\
& =R_{1}\left(0, \theta_{2}\right)-R_{0}\left(0, \theta_{2}\right) \\
& =\theta_{2}\left[\mathrm{E} C(X)-\mathrm{E} C\left(Y_{2}\right)+c_{r 2}\right] .
\end{aligned}
$$

Then, $G_{0}\left(\theta_{1}, \theta_{2}\right)$ is independent of $\theta_{1}$, and decreasing in $\theta_{2}$, taking into account (1). Repeating the earlier argument proves that

$$
\mathrm{E}\left[G_{0}\left(\Theta_{1}, \Theta_{2}\right) \mid D_{1}\left(n_{1}+n_{2}\right)=d_{1}, D_{2}\left(n_{2}\right)=d_{2}\right]
$$

is also decreasing in $d_{1}$ and $d_{2}$; hence so is the expression in (25).

To prove that (24) and (25) are increasing in $n_{2}$, note that both $G_{1}$ and $G_{0}$ are independent of $n_{2}$, and (again following Lemma 2.1) that given $d_{1}$ and $d_{2},\left[\Theta_{1} \mid D_{1}\left(n_{1}+n_{2}\right)=d_{1}\right]$ and $\left[\Theta_{2} \mid D_{2}\left(n_{2}\right)=d_{2}\right]$ are stochastically decreasing in $n_{2}$. Thus, both (26) and (27) are increasing in $n_{2}$; and hence so are (24) and (25).

The proof above also yields the $K$-submodularity of the function $\phi\left(n_{2}, \theta_{1}, \theta_{2}\right)$. 
Corollary 4.5 (i) For $n_{2} \leq N-n_{1}-1, \phi\left(n_{2}, \theta_{1}, \theta_{2}\right)$ is $K_{1}$-submodular with respect to $\left(n_{2}, \theta_{1}\right)$, with

$$
K_{1}=c_{r 1}+\left(c_{r 12}-c_{r 1}-c_{r 2}\right) \theta_{2},
$$

and $K_{2}$-submodular with respect to $\left(n_{2}, \theta_{2}\right)$, with

$$
K_{2}=c_{r 2}+\left(c_{r 12}-c_{r 1}-c_{r 2}\right) \theta_{1} .
$$

(ii) For all $n_{2} \geq N-n_{1}, \phi\left(n_{2}, \theta_{1}, \theta_{2}\right)$ is $K_{1}$-submodular with respect to $\left(n_{2}, \theta_{1}\right)$, with $K_{1}=0$ (i.e., submodular), and $K_{2}$-submodular with respect to $\left(n_{2}, \theta_{2}\right)$, with $K_{2}=c_{r 2}$.

Proof. The claimed properties follow from the increasingness in $\theta_{1}$ and $\theta_{2}$ of $G_{1}\left(\theta_{1}, \theta_{2}\right)$ and $G_{0}\left(\theta_{1}, \theta_{2}\right)$ in the proof of Lemma 4.4, taking into account the following relation:

$$
\begin{aligned}
r\left(\theta_{1}, \theta_{2}\right) & =K_{1} \theta_{1}+c_{r 2} \theta_{2}+c_{i 2} \\
& =K_{2} \theta_{2}+c_{r 1} \theta_{1}+c_{i 2} .
\end{aligned}
$$

\section{Theorem 4.6 For stage 2,}

- If it is optimal to continue inspection in a state $\left(n_{2}, d_{1}, d_{2}\right)$, then it is also optimal to continue inspection in any states $\left(n_{2}, d_{1}^{\prime}, d_{2}^{\prime}\right)$, with $d_{1}^{\prime} \geq d_{1}$ and $d_{2}^{\prime} \geq d_{2}$.

- If it is optimal to stop inspection in a state $\left(n_{2}, d_{1}, d_{2}\right)$, then it is also optimal to stop inspection in any states $\left(n_{2}^{\prime}, d_{1}, d_{2}\right)$, with $n_{2}^{\prime} \geq n_{2}$.

In particular, $\Psi_{n_{2}}\left(d_{1}, d_{2}\right)-\Phi_{n_{2}}\left(d_{1}, d_{2}\right)$ is decreasing in $d_{1}$ and $d_{2}$, and increasing in $n_{2}$.

Proof. Proceed in the same way as in the proof of Theorem 3.2. Specifically, we want to prove via induction that $\Psi_{n_{2}}\left(d_{1}, d_{2}\right)-\Phi_{n_{2}}\left(d_{1}, d_{2}\right)$ is decreasing in $d_{1}$ and $d_{2}$ and increasing in $n_{2}$. Here, in place of (10), we have

$$
\begin{aligned}
& \Psi_{n_{2}}\left(d_{1}, d_{2}\right)-\Phi_{n_{2}}\left(d_{1}, d_{2}\right) \\
= & \mathrm{E}\left[r\left(\Theta_{1}, \Theta_{2}\right) \mid D_{1}\left(n_{1}+n_{2}\right)=d_{1}, D_{2}\left(n_{2}\right)=d_{2}\right]+\Phi_{n_{2}+1}\left(d_{1}+I_{1}, d_{2}+I_{2}\right)-\Phi_{n_{2}}\left(d_{1}, d_{2}\right) \\
& +\mathrm{E}\left[V_{n_{2}+1}\left(d_{1}+I_{1}, d_{2}+I_{2}\right)-\Phi_{n_{2}+1}\left(d_{1}+I_{1}, d_{2}+I_{2}\right)\right]
\end{aligned}
$$

for $n_{2} \leq N-n_{1}-1$, and

$$
\begin{aligned}
& \Psi_{n_{2}}\left(d_{1}, d_{2}\right)-\Phi_{n_{2}}\left(d_{1}, d_{2}\right) \\
= & \mathrm{E}\left[r\left(0, \Theta_{2}\right) \mid D_{2}\left(n_{2}\right)=d_{2}\right]+\Phi_{n_{2}+1}\left(d_{1}+I_{1}, d_{2}+I_{2}\right)-\Phi_{n_{2}}\left(d_{1}, d_{2}\right) \\
& +\mathrm{E}\left[V_{n_{2}+1}\left(d_{1}+I_{1}, d_{2}+I_{2}\right)-\Phi_{n_{2}+1}\left(d_{1}+I_{1}, d_{2}+I_{2}\right)\right]
\end{aligned}
$$


for $n_{2} \geq N-n_{1}$. The rest is the same as in the proof of Theorem 3.2. In particular, the desired monotonicity properties (in $d_{1}, d_{2}$ and $n_{2}$ ) follow from Lemma 4.4 .

Given $n_{1}$, define

$$
S_{n_{1}}\left(n_{2}\right):=\left\{\left(d_{1}, d_{2}\right): 0 \leq d_{1} \leq n_{1}+n_{2}, 0 \leq d_{2} \leq n_{2} ; \Psi_{n_{2}}\left(d_{1}, d_{2}\right)>\Phi_{n_{2}}\left(d_{1}, d_{2}\right)\right\} .
$$

The following properties of $S_{n_{1}}\left(n_{2}\right)$ are direct consequences of Theorem 4.6.

Corollary 4.7 The set $S_{n_{1}}\left(n_{2}\right)$ in (30) satisfies the following properties:

(i) It is a lower set; i.e., $\left(d_{1}, d_{2}\right) \in S_{n_{1}}\left(n_{2}\right) \Rightarrow\left(d_{1}^{\prime}, d_{2}^{\prime}\right) \in S_{n_{1}}\left(n_{2}\right)$, for any $d_{1}^{\prime} \leq d_{1}$ and $d_{2}^{\prime} \leq d_{2}$.

(ii) $S_{n_{1}}\left(n_{2}\right) \subseteq S_{n_{1}}\left(n_{2}^{\prime}\right)$ for any $n_{2}^{\prime} \geq n_{2}$.

In view of (i) above, the boundary of $S_{n_{1}}\left(n_{2}\right)$ is a set of points (two-dimensional vectors), each being a non-dominant point in $S_{n_{1}}\left(n_{2}\right)$, denoted $\left(d_{1}^{*}, d_{2}^{*}\right)$. That is, for any point $\left(d_{1}, d_{2}\right) \in$ $S_{n_{1}}\left(n_{2}\right), d_{1} \geq d_{1}^{*}$ and $d_{2} \geq d_{2}^{*}$ implies $\left(d_{1}, d_{2}\right) \equiv\left(d_{1}^{*}, d_{2}^{*}\right)$.

To summarize, we have

Theorem 4.8 The optimal inspection policy at stage 2 is as follows:

(i) Inspect first the units in $\overline{\mathbf{A}}$, i.e., those that have not yet inspected at stage 1. Inspect the units in $\mathbf{A}$ only after all units in $\overline{\mathbf{A}}$ have been inspected.

(ii) Stop inspection as soon as a state $\left(n_{2}, d_{1}, d_{2}\right)$ with $\left(d_{1}, d_{2}\right) \in S_{n_{1}}\left(n_{2}\right)$ is reached.

To fully characterize the optimal policy at stage 2 , for each $n_{1}$ value (the number of units inspected at stage 1), first compute $\Psi_{n_{2}}\left(d_{1}, d_{2}\right)$ and $\Phi_{n_{2}}\left(d_{1}, d_{2}\right)$ recursively from the expressions presented earlier, (17) through (23). Next, derive the set $S_{n_{1}}\left(n_{2}\right)$. From Corollary 4.7, $S_{n_{1}}\left(n_{2}\right)$ of (30) can be derived as follows: for each $0 \leq d_{2} \leq n_{2}$, derive

$$
d_{1}^{*}\left(d_{2}\right):=\min \left\{d_{1}: \Psi_{n_{2}}\left(d_{1}, d_{2}\right) \leq \Phi_{n_{2}}\left(d_{1}, d_{2}\right)\right\}
$$

then,

$$
S_{n_{1}}\left(n_{2}\right)=\left\{\left(d_{1}, d_{2}\right): 0 \leq d_{2} \leq n_{2}, 0 \leq d_{1}<d_{1}^{*}\left(d_{2}\right)\right\}
$$

\section{A Special Case: Constant Defect Rates}

Here we consider the special case in which both stages have constant defect rates: $\Theta_{1} \equiv \theta_{1}$ and $\Theta_{2} \equiv \theta_{2}$. This special case was studied in some earlier works (e.g., Lindsay and Bishop [11]). Here, in addition to showing that the optimality of either $0 \%$ or $100 \%$ inspection at each 
stage follows directly from our model, we also derive the threshold values that determine the switch-over between inspecting all units or nothing at all.

The optimality of this "all-or-nothing" policy should follow rather intuitively from the constant defect rates. Since in this case there is no "learning" in the sense of updating the conditional defect rate as inspection progresses, if an action is optimal in one state, it should be optimal in all states. In other words, the optimal policy in each stage should be either to inspect all units or not to inspect any unit.

Consider first the optimal policy at stage 2 , given that $n_{1}$ units have been inspected at stage 1. Then, the total expected cost at stage 2 given $n_{2}$ units are inspected can be expressed as follows:

$$
\begin{aligned}
& n_{2} R_{1}\left(\theta_{1}, \theta_{2}\right)+n_{1} R_{0}\left(0, \theta_{2}\right)+\left(N-n_{1}-n_{2}\right) R_{0}\left(\theta_{1}, \theta_{2}\right) \\
= & n_{2}\left[R_{1}\left(\theta_{1}, \theta_{2}\right)-R_{0}\left(\theta_{1}, \theta_{2}\right)\right]+n_{1} R_{0}\left(0, \theta_{2}\right)+\left(N-n_{1}\right) R_{0}\left(\theta_{1}, \theta_{2}\right),
\end{aligned}
$$

for $n_{2} \leq N-n_{1}$, and

$$
\begin{aligned}
& \left(N-n_{1}\right) R_{1}\left(\theta_{1}, \theta_{2}\right)+\left(n_{1}+n_{2}-N\right) R_{1}\left(0, \theta_{2}\right)+\left(N-n_{2}\right) R_{0}\left(0,, \theta_{2}\right) \\
= & n_{2}\left[R_{1}\left(0, \theta_{2}\right)-R_{0}\left(0, \theta_{2}\right)\right]+\left(N-n_{1}\right)\left[R_{1}\left(\theta_{1}, \theta_{2}\right)-R_{1}\left(0, \theta_{2}\right)\right]+N R_{0}\left(0, \theta_{2}\right)
\end{aligned}
$$

for $N-n_{1} \leq n_{2} \leq N$. Hence, to minimize the above costs, the solution is:

- $n_{2}^{*}=0$, if $R_{1}\left(\theta_{1}, \theta_{2}\right)>R_{0}\left(\theta_{1}, \theta_{2}\right)$,

- $n_{2}^{*}=N$, if $R_{1}\left(0, \theta_{2}\right) \leq R_{0}\left(0, \theta_{2}\right)$,

- $n_{2}^{*}=N-n_{1}$, if $R_{1}\left(\theta_{1}, \theta_{2}\right) \leq R_{0}\left(\theta_{1}, \theta_{2}\right)$ and $R_{1}\left(0, \theta_{2}\right)>R_{0}\left(0, \theta_{2}\right)$.

From (12) and (13), it is easy to verify that $R_{1}\left(0, \theta_{2}\right) \leq R_{0}\left(0, \theta_{2}\right)$ is equivalent to

$$
\theta_{2} \geq \frac{c_{i 2}}{\mathrm{E} C\left(Y_{2}\right)-\mathrm{E} C(X)-c_{r 2}}:=\bar{\theta}_{2}
$$

and $R_{1}\left(\theta_{1}, \theta_{2}\right)>R_{0}\left(\theta_{1}, \theta_{2}\right)$ is equivalent to

$$
\theta_{2}<\frac{c_{i 2}-\theta_{1}\left[\mathrm{E} C\left(Y_{1}\right)-\mathrm{E} C(X)-c_{r 1}\right]}{\theta_{1}\left[\mathrm{E} C\left(Y_{12}\right)-\mathrm{E} C\left(Y_{1}\right)-c_{r 12}+c_{r 1}\right]+\left(1-\theta_{1}\right)\left[\mathrm{E} C\left(Y_{2}\right)-\mathrm{E} C(X)-c_{r 2}\right]}:=\underline{\theta}_{2} .
$$

Summing up the above discussion, we have

Lemma 5.1 Suppose both stages have constant defect rates: $\Theta_{1} \equiv \theta_{1}$ and $\Theta_{2} \equiv \theta_{2}$. Suppose stage 1 has inspected a total of $n_{1}=|\mathbf{A}|$ units. Then, the optimal policy at stage 2 is:

(i) to inspect all $N$ units, if $\theta_{2} \geq \bar{\theta}_{2}$ in (35); 
(ii) not to inspect at all, if $\theta_{2}<\underline{\theta}_{2}$ in (36); and

(iii) to inspect the $N-n_{1}$ units in $\overline{\mathbf{A}}$ only, if $\bar{\theta}_{2}>\theta_{2} \geq \underline{\theta}_{2}$.

Proof. The only point left out of the above discussion is that in (iii) we need to justify that $\bar{\theta}_{2} \geq \underline{\theta}_{2}$. But this can be directly verified from comparing (35) and (36), along with (1) and (2), taking into account that $\bar{\theta}_{2} \leq 1$.

The optimal costs corresponding to the three cases in the above lemma are, respectively:

$$
\begin{aligned}
& \phi_{1}=n_{1} R_{1}\left(0, \theta_{2}\right)+\left(N-n_{1}\right) R_{1}\left(\theta_{1}, \theta_{2}\right), \\
& \phi_{2}=n_{1} R_{0}\left(0, \theta_{2}\right)+\left(N-n_{1}\right) R_{0}\left(\theta_{1}, \theta_{2}\right), \\
& \phi_{3}=n_{1} R_{0}\left(0, \theta_{2}\right)+\left(N-n_{1}\right) R_{1}\left(\theta_{1}, \theta_{2}\right) .
\end{aligned}
$$

Hence, the total expected cost at stage 1 , given $n_{1}$ units are inspected, can be expressed as

$$
n_{1}\left(c_{i 1}+\theta_{1} c_{r 1}\right)+\phi^{*}\left(n_{1}, \theta_{1}, \theta_{2}\right)
$$

where $\phi^{*}$ is equal to $\phi_{1}, \phi_{2}$ or $\phi_{3}$ according to which range $\theta_{2}$ falls into in Lemma 5.1. Regardless, however, $\phi^{*}$ is linear in $n_{1}$. Hence, the expression in (40) is also a linear function of $n_{1}$; and the optimal $n_{1}$ that minimizes it is either zero or $N$. Hence, we only need to compare these two alternatives. Observe from the above discussion that at stage 1, inspecting $N$ units is better than inspecting 0 unit if and only if

$$
N\left(c_{i 1}+\theta_{1} c_{r 1}\right)+\min \left\{N R_{1}\left(0, \theta_{2}\right), N R_{0}\left(0, \theta_{2}\right)\right\} \leq \min \left\{N R_{1}\left(\theta_{1}, \theta_{2}\right), N R_{0}\left(\theta_{1}, \theta_{2}\right)\right\}
$$

The above is equivalent to:

$$
\begin{aligned}
& R_{1}\left(\theta_{1}, \theta_{2}\right)-\theta_{1} c_{r 1} \geq c_{i 1}+\min \left\{R_{1}\left(0, \theta_{2}\right), R_{0}\left(0, \theta_{2}\right)\right\}, \\
& R_{0}\left(\theta_{1}, \theta_{2}\right)-\theta_{1} c_{r 1} \geq c_{i 1}+\min \left\{R_{1}\left(0, \theta_{2}\right), R_{0}\left(0, \theta_{2}\right)\right\} .
\end{aligned}
$$

Now, subtract $R_{1}\left(0, \theta_{2}\right)$ from both sides of (41). The left hand side then becomes

$$
\begin{aligned}
& R_{1}\left(\theta_{1}, \theta_{2}\right)-R_{1}\left(0, \theta_{2}\right)-\theta_{1} c_{r 1} \\
= & {\left[R_{1}\left(1, \theta_{2}\right)-R_{1}\left(0, \theta_{2}\right)-c_{r 1}\right] \theta_{1}, }
\end{aligned}
$$

taking into account (12). Also note that the quantity in the squared brackets above is nonnegative. Hence, from (41), we obtain

$$
\theta_{1} \geq \frac{c_{i 1}+\min \left\{R_{0}\left(0, \theta_{2}\right)-R_{1}\left(0, \theta_{2}\right), 0\right\}}{R_{1}\left(1, \theta_{2}\right)-R_{1}\left(0, \theta_{2}\right)-c_{r 1}}:=\theta^{\prime}
$$


Note that when the denominator above is equal to zero, $\theta^{\prime}$ is understood to be 1 . Similarly, from (42), we obtain

$$
\theta_{1} \geq \frac{c_{i 1}+\min \left\{R_{1}\left(0, \theta_{2}\right)-R_{0}\left(0, \theta_{2}\right), 0\right\}}{R_{0}\left(1, \theta_{2}\right)-R_{0}\left(0, \theta_{2}\right)-c_{r 1}}:=\theta^{\prime \prime}
$$

with the understanding that $\theta^{\prime \prime}=1$ should the denominator above become zero.

Therefore, the threshold value at stage 1 should be set at $\theta_{1}^{*}=\max \left\{\theta^{\prime}, \theta^{\prime \prime}\right\}$ : inspect all $N$ units if $\theta_{1} \geq \theta_{1}^{*}$; otherwise, inspect nothing. Note that if the denominator in either (43) or (44) is equal to zero, then inspecting $N$ units only if $\theta_{1} \equiv 1$.

Once the optimal decision at stage 1 is carried out, it impacts the decision at stage 2 following Lemma 5.1. Specifically, part (iii) of the lemma should be combined into the first two parts as follows: If stage 1 inspects all $N$ units, i.e., $n_{1}=|\mathbf{A}|=N$, then it is optimal to inspect all $N$ units if $\theta_{2} \geq \underline{\theta}_{2}$, and inspect 0 unit if $\theta_{2}<\underline{\theta}_{2}$. If $n_{1}=|\mathbf{A}|=0$, then it is optimal to inspect all $N$ units if $\theta_{2} \geq \bar{\theta}_{2}$, and inspect 0 unit if $\theta_{2}<\bar{\theta}_{2}$.

To summarize, we have

Theorem 5.2 Suppose both stages have constant defect rates: $\Theta_{1} \equiv \theta_{1}$ and $\Theta_{2} \equiv \theta_{2}$.

(i) At stage 1, it is optimal to inspect all $N$ units if $\theta_{1} \geq \theta_{1}^{*}$, and inspect 0 unit if $\theta_{1}<\theta_{1}^{*}$, where $\theta_{1}^{*}=\max \left\{\theta^{\prime}, \theta^{\prime \prime}\right\}$, with $\theta^{\prime}, \theta^{\prime \prime}$ following $(43,44)$.

(ii) At stage 2, it is optimal to inspect all $N$ units if $\theta_{2} \geq \theta_{2}^{*}$, and inspect 0 unit if $\theta_{2}<\theta_{2}^{*}$, where $\theta_{2}^{*}=\underline{\theta}_{2}$ or $\bar{\theta}_{2}$, according to, respectively, $\theta_{1} \geq \theta_{1}^{*}$ or $\theta_{1}<\theta_{1}^{*}$, with $\bar{\theta}_{2}$ and $\underline{\theta}_{2}$ following $(35,36)$.

\section{Optimal Policy at Stage 1}

We now revisit the characterization of the optimal policy at stage 1 . Recall in $\S 2.3$ we have established, under Condition 2.2, that there exists an optimal policy at stage 1 with the threshold structure described in Theorem 3.3. Here, let us examine more closely the derivation of the optimal policy at stage 1 via dynamic programming as outlined in $\S 3$. The starting point is to compute the function in (5), which we rewrite as follows, recovering the stage indices:

$$
\Phi_{n_{1}}\left(d_{1}\right)=\mathrm{E}\left[\phi\left(n_{1}, \Theta_{1}\right) \mid D_{1}\left(n_{1}\right)=d_{1}\right],
$$

which, in turn, is equal to the optimal value at stage 2 : $V_{0}\left(D_{1}\left(n_{1}\right), 0\right)$, with $D_{1}\left(n_{1}\right)=d_{1}$; refer to the dynamic programming formulation of the stage 2 problem in $\S 4.2$ (preceding Lemma 4.4). In other words, for each $\left(d_{1}, n_{1}\right)$, with $d_{1} \leq n_{1} \leq N$, we first solve the stage 2 problem, 
which returns the functional value in (5). Then, we go through the recursion in (5) through (7) to generate the optimal actions in stage 1 for each state $\left(d_{1}, n_{1}\right)$.

The above procedure applies, with or without Condition 2.2. In other words, even without Condition 2.2, the dynamic programming problem for stage 1 is already significantly benefited from the structure of the optimal policy at stage 2. With Condition 2.2, there is an additional advantage in the dynamic programming recursion: for each $n$, as we increase the $d$ value and compare $\Psi_{n}(d)$ with $\Phi_{n}(d)$, we can stop as soon as $\Psi_{n}(d) \leq \Phi_{n}(d)$ (which will continue to hold for larger $d$ values, as guaranteed by the threshold structure). This reduces the computational effort by roughly half.

Also note that Condition 2.2 is only a sufficient condition: without it, the optimal actions may still follow a threshold structure. In general, the convexity and submodularity properties required in Condition 2.2 need not be satisfied. Below, however, we show that these properties are indeed satisfied in a special case: when the defect rate at stage 2 is a constant.

Theorem 6.1 Suppose stage 2 has a constant defect rate: $\Theta_{2} \equiv \theta_{2}$. Then Condition 2.2 is satisfied. Specifically, the function $\phi\left(n_{1}, \theta_{1}\right)$ (optimal cost at stage 2 , given $n_{1}$ units are inspected at stage 1 and $\left.\Theta_{1}=\theta_{1}\right)$ is linear in $n_{1}$, and $K$-submodular in $\left(n_{1}, \theta_{1}\right)$ with $K=c_{r 1}$. Consequently, the optimal policy at stage 1 has the threshold structure described in Theorem 3.3 .

Proof. Under the stated conditions, $\phi\left(n_{1}, \theta_{1}\right)$ is just the $\phi^{*}$ function following Lemma 5.1. Hence the linearity in $n_{1}$ follows from the discussion there.

For $K$-submodularity, we want to show that for $\theta_{1}^{\prime} \geq \theta_{1}$,

$$
\text { LHS }:=\left[\phi\left(n_{1}+1, \theta_{1}\right)+\phi\left(n_{1}, \theta_{1}^{\prime}\right)\right]-\left[\phi\left(n_{1}, \theta_{1}\right)+\phi\left(n_{1}+1, \theta_{1}^{\prime}\right)\right] \geq c_{r 1}\left(\theta_{1}^{\prime}-\theta_{1}\right) .
$$

Consider the three cases in Lemma 5.1.

Case (i). The optimal solution is $\phi_{1}$ at $\theta_{1}$, and it is also optimal at $\theta_{1}^{\prime}$. Hence, following (37), we have

$$
\begin{aligned}
\mathrm{LHS} & =R_{1}\left(\theta_{1}^{\prime}, \theta_{2}\right)-R_{1}\left(\theta_{1}, \theta_{2}\right) \\
& =c_{r 1}\left(\theta_{1}^{\prime}-\theta_{1}\right)+\left(c_{r 12}-c_{r 1}-c_{r 2}\right)\left(\theta_{1}^{\prime}-\theta_{1}\right) \theta_{2} \\
& \geq c_{r 1}\left(\theta_{1}^{\prime}-\theta_{1}\right)
\end{aligned}
$$

where the inequality follows from the fact that $c_{r 12}-c_{r 1}-c_{r 2} \geq 0$.

Case (ii). At $\theta_{1}$, the optimal solution is $\phi_{2}$; whereas at $\theta_{1}^{\prime}$, the optimal solution could be either $\phi_{2}$ or $\phi_{3}$, since increasing $\theta_{1}$ decreases the threshold value in (36). 
First suppose at $\theta_{1}^{\prime}, \phi_{2}$ is optimal. Then, from (38), we have

$$
\begin{aligned}
\mathrm{LHS} & =R_{0}\left(\theta_{1}^{\prime}, \theta_{2}\right)-R_{0}\left(\theta_{1}, \theta_{2}\right) \\
& =\left(\theta_{1}^{\prime}-\theta_{1}\right)\left[\mathrm{E} C\left(Y_{1}\right)-\mathrm{E} C(X)\right]+\theta_{2}\left(\theta_{1}^{\prime}-\theta_{1}\right)\left[\mathrm{E} C\left(Y_{12}\right)+\mathrm{E} C(X)-\mathrm{E} C\left(Y_{1}\right)-\mathrm{E} C\left(Y_{2}\right)\right] \\
& \geq\left(\theta_{1}^{\prime}-\theta_{1}\right) c_{r 1},
\end{aligned}
$$

where the last inequality follows from (1) and (4).

Next, suppose at $\theta_{1}^{\prime}, \phi_{3}$ is optimal. Then, from (38) and (39), we have

$$
\begin{aligned}
\mathrm{LHS} & =R_{1}\left(\theta_{1}^{\prime}, \theta_{2}\right)-R_{0}\left(\theta_{1}, \theta_{2}\right) \\
& \geq R_{1}\left(\theta_{1}^{\prime}, \theta_{2}\right)-R_{1}\left(\theta_{1}, \theta_{2}\right) \\
& =c_{r 1}\left(\theta_{1}^{\prime}-\theta_{1}\right)+\left(c_{r 12}-c_{r 1}-c_{r 2}\right)\left(\theta_{1}^{\prime}-\theta_{1}\right) \theta_{2} \\
& \geq c_{r 1}\left(\theta_{1}^{\prime}-\theta_{1}\right)
\end{aligned}
$$

where the first inequality follows from $\phi_{2} \leq \phi_{3}$, since $\phi_{2}$ is optimal at $\theta_{1}$ [cf. (38), (39)]. Case (iii). The optimal solution is $\phi_{3}$ at $\theta_{1}$. It is also optimal at $\theta_{1}^{\prime}$, since increasing $\theta_{1}$ only decreases the lower threshold value - the right hand side in (36). Hence, following (39), we have, same as in Case (i),

$$
\begin{aligned}
\mathrm{LHS} & =R_{1}\left(\theta_{1}^{\prime}, \theta_{2}\right)-R_{1}\left(\theta_{1}, \theta_{2}\right) \\
& =c_{r 1}\left(\theta_{1}^{\prime}-\theta_{1}\right)+\left(c_{r 12}-c_{r 1}-c_{r 2}\right)\left(\theta_{1}^{\prime}-\theta_{1}\right) \theta_{2} \\
& \geq c_{r 1}\left(\theta_{1}^{\prime}-\theta_{1}\right) .
\end{aligned}
$$

Note that when $\Theta_{2} \equiv \theta_{2}, d_{1}^{*}\left(d_{2}\right)$ in (31) is independent of $d_{2}$, since both $\Psi_{n_{2}}\left(d_{1}, d_{2}\right)$ and $\Phi_{n_{2}}\left(d_{1}, d_{2}\right)$ are independent of $d_{2}$. Hence, we have

Corollary 6.2 Suppose stage 2 has a constant defect rate. Then,

(i) for stage 1 the policy described in Theorem 3.3 is optimal;

(ii) for stage 2, given $|\mathbf{A}|=n_{1}$, there exists a $d_{n_{1}, n_{2}}$ for each $n_{2} \leq N$, such that it is optimal to stop inspection at $\left(n_{2}, d_{1}, d_{2}\right)$ if and only if $d_{1}<d_{n_{1}, n_{2}}$.

Based on the above Corollary, we can develop a heuristic policy as follows: First, assume that stage 2 has a constant defect rate: $\Theta_{2} \equiv \mathrm{E}\left[\Theta_{2}\right]$, and derive a threshold policy for stage 1 as in Theorem 3.3; next, for stage 2, still treat the defect rate as what it is, i.e., a random variable, and derive the optimal threshold policy following Theorem 4.8. 


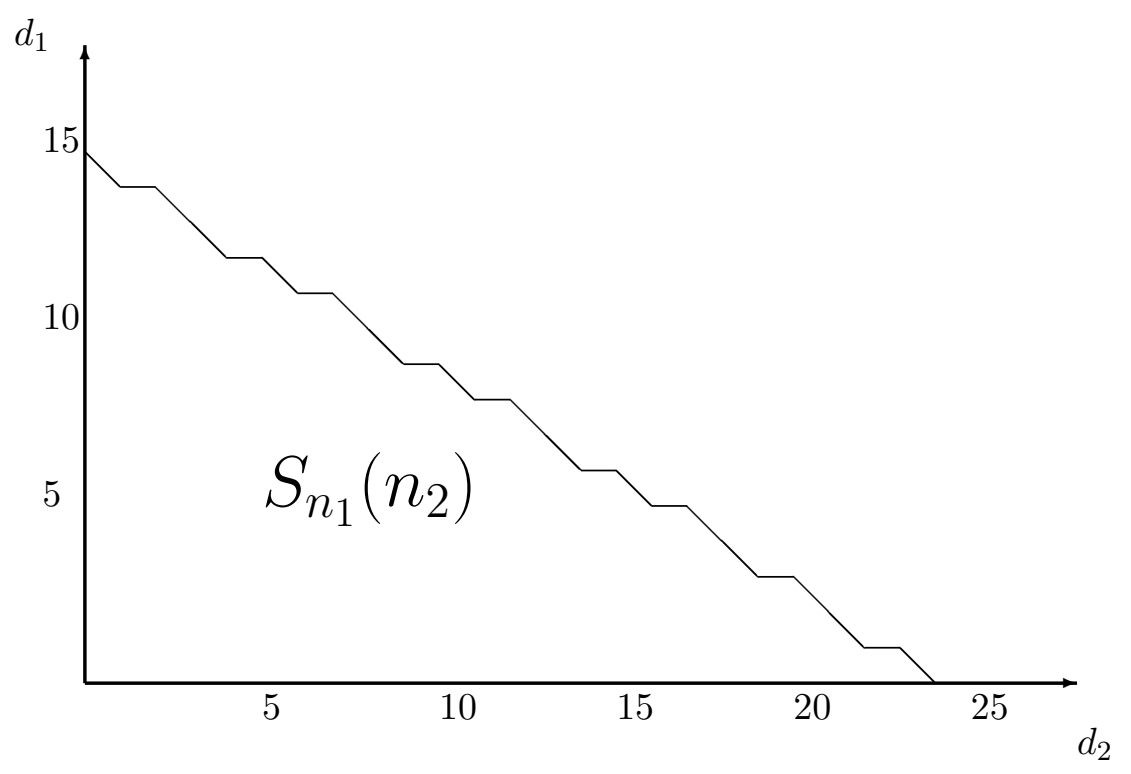

Figure 1: Threshold structure at stage 2

Below, we study this heuristic policy in two examples, and compare its performance against the optimal policy. In addition, we also compare it against the solution obtained by treating the defect rates at both stages as constant; i.e., assuming $\Theta_{1} \equiv \mathrm{E}\left[\Theta_{1}\right]$, and $\Theta_{2} \equiv \mathrm{E}\left[\Theta_{2}\right]$.

Example 6.3 Suppose $N=30 ; c_{i 1}=6.5, c_{i 2}=13.5 ; c_{r 1}=1.0, c_{r 2}=2.0, c_{r 12}=6.0$; $\mathrm{E} C(X)=3.5, \mathrm{E} C\left(Y_{1}\right)=18.5, \mathrm{E} C\left(Y_{2}\right)=20, \mathrm{E} C\left(Y_{12}\right)=35.5 ; \Theta_{1}$ and $\Theta_{2}$ are both uniformly distributed on $(0,1)$. Note that inequality (1), (2) and (4) are all satisfied in this case. The results under the different policies mentioned above are summarized below:

(i) Follow the optimal policy, which takes the following form: At stage 1, stop inspection in $\left(n_{1}, d_{1}\right)$ if and only if $d_{1}<d_{n_{1}}$, with $d_{n_{1}}$ taking the following values:

\begin{tabular}{|l|l|l|l|l|l|l|l|l|l|l|l|l|l|l|l|}
\hline$n_{1}$ & 0 & 1 & 2 & 3 & 4 & 5 & 6 & 7 & 8 & 9 & 10 & 11 & 12 & 13 & 14 \\
\hline$d_{n_{1}}$ & 0 & 1 & 1 & 2 & 2 & 3 & 4 & 4 & 5 & 5 & 6 & 7 & 7 & 8 & 8 \\
\hline \hline$n_{1}$ & 15 & 16 & 17 & 18 & 19 & 20 & 21 & 22 & 23 & 24 & 25 & 26 & 27 & 28 & 29 \\
\hline$d_{n_{1}}$ & 9 & 10 & 10 & 11 & 11 & 12 & 13 & 13 & 13 & 14 & 14 & 15 & 15 & 15 & 14 \\
\hline
\end{tabular}

At stage 2, for each $\left(n_{1}, n_{2}\right)$, stop inspection if and only if $\left(d_{1}, d_{2}\right) \in S_{n_{1}}\left(n_{2}\right)$, where $S_{n_{1}}\left(n_{2}\right)$ follows the specification at the end of $\S 4$. An illustration of $S_{n_{1}}\left(n_{2}\right)$, for $n_{1}=10$ and $n_{2}=15$, is given in Figure 1. The expected total cost under this optimal policy is 513.1.

(ii) Follow the heuristic policy specified above: At stage 1, treating the defect rate at stage 2 as a constant, the optimal policy takes the form of stopping inspection at $\left(n_{1}, d_{1}\right)$ if and only if $d_{1}<d_{n_{1}}$, with the threshold values $d_{n_{1}}$ given below: 


\begin{tabular}{|l|l|l|l|l|l|l|l|l|l|l|l|l|l|l|l|}
\hline$n_{1}$ & 0 & 1 & 2 & 3 & 4 & 5 & 6 & 7 & 8 & 9 & 10 & 11 & 12 & 13 & 14 \\
\hline$d_{n_{1}}$ & 0 & 0 & 0 & 1 & 1 & 2 & 2 & 3 & 3 & 4 & 4 & 4 & 5 & 5 & 6 \\
\hline \hline$n_{1}$ & 15 & 16 & 17 & 18 & 19 & 20 & 21 & 22 & 23 & 24 & 25 & 26 & 27 & 28 & 29 \\
\hline$d_{n_{1}}$ & 6 & 7 & 7 & 8 & 8 & 9 & 9 & 10 & 10 & 11 & 11 & 12 & 12 & 13 & 14 \\
\hline
\end{tabular}

At stage 2, we follow the optimal threshold policy in Theorem 4.8, which is the same as the stage-2 policy in (i) above. Under this policy, the expected total cost is 524.5 , less than $3 \%$ above the optimal cost.

(iv) Treat the defect rates at both stages as constants. Following Theorem 5.2, this results in a policy that inspects all units in stage 1 , and inspect 0 unit in stage 2 ; and the expected total cost is 562.5 , about $10 \%$ above the optimal cost.

Example 6.4 Consider a variation of the above example, with $N=30 ; c_{i 1}=3.5, c_{i 2}=7.5$; $c_{r 1}=1.0, c_{r 2}=2.0, c_{r 12}=3.5 ; \mathrm{E} C(X)=3.5, \mathrm{E} C\left(Y_{1}\right)=13.5, \mathrm{E} C\left(Y_{2}\right)=15, \mathrm{E} C\left(Y_{12}\right)=25.5 ;$ $\Theta_{1}$ and $\Theta_{2}$ are both uniformly distributed on $(0,1)$. The expected total costs are: 357.3 for optimal policy; 360.5 for the heuristic policy (less than 1\% above the optimal cost); and 378.8 for the policy that treats both defect rates as constants ( $6 \%$ above the optimal cost).

From the above examples, it should be clear that the optimal policy and the heuristic policy are exactly the same at stage 2 : both involve (the same) $N^{2}$ threshold curves, such as the one illustrated in Figure 1. Each such curve requires no more than $N^{2}$ steps to generate. Hence, the overall computational effort is $O\left(N^{4}\right)$ to generate the policy at stage 2. At stage 1, as discussed at the beginning of the section, the optimal policy can be derived via the dynamic programming recursion, with a computational effort of $O\left(N^{2}\right)$; while the heuristic policy can be derived using roughly half of that effort, thanks to its guaranteed threshold structure, with $N$ thresholds.

For implementation, both policies can be pre-computed off line. The inspection is then carried out on line: proceed from stage 1 to stage 2 ; at either stage, inspect one unit at a time, and check the number of identified defects against the precomputed threshold values, and stop inspection once the number falls below the threshold. Furthermore, at stage 2, always inspect first those units that have not been inspected at stage 1 . Note, however, that for the optimal policy the threshold structure at stage 1 is not guaranteed. Hence, in those cases where the optimal policy does not have a threshold structure at stage 1 , the heuristic policy has the additional advantage of being easier to implement. 


\section{General Cost Functions}

Our results developed above are readily extended to a more general cost function (corresponding to warranties or service contracts). For instance, as in [6], we can allow $C(T)$ to be the (warranty) cost function for the whole batch, with $T$ denoting the total (cumulative) lifetime of all $N$ units in the batch. For instance,

$$
C(T)=(c N)[N W-T]^{+} /(N W)=c[N-T / W]^{+},
$$

where $c>0$ is the selling price of each unit, $W$ is the guaranteed total lifetime of the $N$ units, and $[x]^{+}$denotes $\max \{x, 0\}$. In this "cumulative warranty" model $([4,14])$, the manufacturer pays back part of the selling price on a pro rata basis. This type of warranty applies mostly to reliability systems, where spare parts (in cold stand-by) are used extensively.

Below we shall focus on stage 2 , and show that the structure of the optimal policy in $\S 4$ remains valid under the general cost function as described above. As to stage 1, the comments at the beginning of $\S 6$ still apply.

Consider the optimal policy at stage 2 . Same as in $\S 4$, suppose the batch has already been inspected at stage 1, with the inspected items identified as set $\mathbf{A}$. Recall, the lifetimes of the items follow the distributions of $X, Y_{1}, Y_{2}$, and $Y_{12}$, respectively for good items and items that are stage 1 , stage 2 , and 1-2 defects. Assume $X \geq_{\text {st }} Y_{j} \geq_{\text {st }} Y_{12}$ for $j=1,2$; all lifetimes are independent; and that $C(T)$ is a convex (and decreasing) function of $T$.

Let $Z\left(\theta_{1}, \theta_{2}\right)$ denote the random variable which is equal to $X, Y_{1}, Y_{2}$ and $Y_{12}$ with probability $\left(1-\theta_{1}\right)\left(1-\theta_{2}\right), \theta_{1}\left(1-\theta_{2}\right),\left(1-\theta_{1}\right) \theta_{2}$ and $\theta_{1} \theta_{2}$, respectively. That is, $Z\left(\theta_{1}, \theta_{2}\right)$ is the lifetime of a unit which is not inspected at either stage, given the defect rates at the two stages are $\theta_{1}$ and $\theta_{2}$.

For convenience in notation, write

$$
Z_{n, m}\left(\theta_{1}, \theta_{2}\right):=\sum_{i=n}^{m} Z_{i}\left(\theta_{1}, \theta_{2}\right), \quad X_{n, m}:=\sum_{i=n}^{m} X_{i}
$$

where $X_{i}$ are iid samples of $X$, and $Z_{i}\left(\theta_{1}, \theta_{2}\right)$ are iid samples of $Z\left(\theta_{1}, \theta_{2}\right)$.

In the same spirit as (1) and (2), we assume

$$
\mathrm{E} C\left(X_{1, N-1}+Y_{1}\right)-\mathrm{E} C\left(X_{1, N}\right) \geq c_{r 1}, \quad \mathrm{E} C\left(X_{1, N-1}+Y_{2}\right)-\mathrm{E} C\left(X_{1, N}\right) \geq c_{r 2},
$$

and

$$
\begin{aligned}
& \mathrm{E} C\left(X_{1, N-1}+Y_{12}\right)-\mathrm{E} C\left(X_{1, N-1}+Y_{1}\right) \geq c_{r 12}-c_{r 1}, \\
& \mathrm{E} C\left(X_{1, N-1}+Y_{12}\right)-\mathrm{E} C\left(X_{1, N-1}+Y_{2}\right) \geq c_{r 12}-c_{r 2} .
\end{aligned}
$$


Same as before, let $\mathbf{A}$ denote the set of items that have been inspected at stage 1, and let $\overline{\mathbf{A}}$ be its complement. Suppose $|\mathbf{A}|=n_{1}$, and suppose stage 2 has inspected $n_{2}$ items from $\mathbf{A}$ and $\bar{n}_{2}$ items from $\overline{\mathbf{A}}$. If stage 2 stops inspection at this point and given $\Theta_{1}=\theta_{1}$ and $\Theta_{2}=\theta_{2}$, then the expected warranty cost is

$$
\phi\left(n_{2}, \bar{n}_{2}, \theta_{1}, \theta_{2}\right)=\mathrm{E}\left[C\left(X_{1, n_{2}+\bar{n}_{2}}+Z_{n_{2}+\bar{n}_{2}+1, n_{1}+\bar{n}_{2}}\left(0, \theta_{2}\right)+Z_{n_{1}+\bar{n}_{2}+1, N}\left(\theta_{1}, \theta_{2}\right)\right] .\right.
$$

We first examine the priority structure in Theorem 4.2. As in Section $\S 4.1$, let $V\left(n_{2}, \bar{n}_{2}, d_{1}, d_{2}\right)$ denote the optimal expected future cost, including inspection and rework cost and the total warranty cost, starting from the state $\left(n_{2}, \bar{n}_{2}, d_{1}, d_{2}\right)$. Similar to (14), we need to show

$$
\begin{array}{ll} 
& \mathrm{E}\left[V\left(n_{2}+1, \bar{n}_{2}, d_{1}, d_{2}+I_{2}^{\prime}\right)\right]-\mathrm{E}\left[V\left(n_{2}, \bar{n}_{2}+1, d_{1}+I_{1}, d_{2}+I_{2}\right)\right] \\
\geq \quad & \mathrm{E}\left[r\left(\Theta_{1}, \Theta_{2}\right) \mid D_{1}\left(n_{1}+\bar{n}_{2}\right)=d_{1}, D_{2}\left(n_{2}+\bar{n}_{2}\right)=d_{2}\right] \\
& -\mathrm{E}\left[r\left(0, \Theta_{2}\right) \mid D_{2}\left(n_{2}+\bar{n}_{2}\right)=d_{2}\right] .
\end{array}
$$

The only modification needed in the proof concerns the inequalities involved in the initial step of the induction (when $n_{2}+\bar{n}_{2}=N-2$ ) and in the step of $B-b \geq \Delta$. Since the two modifications are similar, we illustrate the latter only. The inequality in question here (omitting the conditioning) is

$$
\phi\left(n_{2}+1, \bar{n}_{2}, \theta_{1}, \theta_{2}\right)-\phi\left(n_{2}, \bar{n}_{2}+1, \theta_{1}, \theta_{2}\right) \geq r\left(\theta_{1}, \theta_{2}\right)-r\left(0, \theta_{2}\right) .
$$

Let

$$
\eta:=X_{1, n_{2}+\bar{n}_{2}+1}+Z_{n_{2}+\bar{n}_{2}+2, n_{1}+\bar{n}_{2}}\left(0, \theta_{2}\right)+Z_{n_{1}+\bar{n}_{2}+2, N}\left(\theta_{1}, \theta_{2}\right) .
$$

Then, the left hand side of the last inequality

$$
\begin{aligned}
& \phi\left(n_{2}+1, \bar{n}_{2}, \theta_{1}, \theta_{2}\right)-\phi\left(n_{2}, \bar{n}_{2}+1, \theta_{1}, \theta_{2}\right) \\
= & \mathrm{E} C\left(\eta+Z\left(\theta_{1}, \theta_{2}\right)\right)-\mathrm{E} C\left(\eta+Z\left(0, \theta_{2}\right)\right) \\
= & \theta_{1}\left(1-\theta_{2}\right)\left[\mathrm{E} C\left(\eta+Y_{1}\right)-\mathrm{E} C(\eta+X)\right] \\
& +\theta_{1} \theta_{2}\left[\mathrm{E} C\left(\eta+Y_{12}\right)-\mathrm{E} C\left(\eta+Y_{2}\right)\right] \\
\geq & \theta_{1}\left(1-\theta_{2}\right)\left[\mathrm{E} C\left(X_{1, N-1}+Y_{1}\right)-\mathrm{E} C\left(X_{1, N-1}+X\right)\right] \\
& +\theta_{1} \theta_{2}\left[\mathrm{E} C\left(X_{1, N-1}+Y_{12}\right)-\mathrm{E} C\left(X_{1, N-1}+Y_{2}\right)\right] \\
\geq & c_{r 1} \theta_{1}\left(1-\theta_{2}\right)+\left(c_{r 12}-c_{r 2}\right) \theta_{1} \theta_{2} \\
= & r\left(\theta_{1}, \theta_{2}\right)-r\left(0, \theta_{2}\right) .
\end{aligned}
$$

Note that the first inequality above follows from the decreasing convexity of the function $C(\cdot)$, taking into account $\eta \leq_{\mathrm{st}} X_{1, N-1}$ (also see the proof of Lemma 7.1 below). The second inequality follows from (45) and (47). 
Therefore, we can follow the state definition of $\S 4.2$. In particular, if $\Theta_{1}=\theta_{1}, \Theta_{2}=\theta_{2}$, and we stop inspection after inspecting $n_{2}$ units at stage 2 , then the expected warranty cost is:

$$
\phi\left(n_{2}, \theta_{1}, \theta_{2}\right):=\mathrm{E} C\left(X_{1, n_{2}}+Z_{n_{2}+1, n_{1}+n_{2}}\left(0, \theta_{2}\right)+Z_{n_{1}+n_{2}+1, N}\left(\theta_{1}, \theta_{2}\right)\right)
$$

for $n_{2} \leq N-n_{1}-1$ and

$$
\phi\left(n_{2}, \theta_{1}, \theta_{2}\right):=\mathrm{E} C\left(X_{1, n_{2}}+Z_{n_{2}+1, N}\left(0, \theta_{2}\right)\right)
$$

for $n_{2} \geq N-n_{1}$.

Substituting $\phi(\cdot)$ of (49) and (50) above into (20) yield the problem formulation under the general cost function.

Lemma 7.1 Suppose the (warranty) cost function of the whole batch, $C(\cdot)$, is a decreasing and convex function. Then, given $\theta_{1}$ and $\theta_{2}, \phi\left(n_{2}, \theta_{1}, \theta_{2}\right)$ defined in (49) and (50) above is decreasing and convex in $n_{2}$.

Proof. Since $\theta_{1}$ and $\theta_{2}$ are given here, write $\phi\left(n_{2}, \theta_{1}, \theta_{2}\right)$ as $\phi\left(n_{2}\right)$ for simplicity. Clearly, following the assumed stochastic orderings among the lifetimes, we have

$$
X \geq_{\mathrm{st}} Z\left(0, \theta_{2}\right) \geq_{\mathrm{st}} Z\left(\theta_{1}, \theta_{2}\right) .
$$

for any $\theta_{1}$ and $\theta_{2}$. Since $C(\cdot)$ is a decreasing function, $\phi\left(n_{2}\right)$ is decreasing in $n_{2}$, following the expressions in (49) and (50).

To prove convexity, we use coupling. Consider first the case of $n_{2} \leq N-n_{1}-1$. Since $X \geq_{\text {st }} Z\left(\theta_{1}, \theta_{2}\right)$, we can have, for $j=1,2, X^{j} \geq Z^{j}$ almost surely (a.s.), with $X^{j}$ and $Z^{j}$ equal in distribution to $X$ and $Z\left(\theta_{1}, \theta_{2}\right)$, respectively(while maintaining the independence between $X^{1}$ and $X^{2}$ and between $Z^{1}$ and $\left.Z^{2}\right)$. Let $\tau$ be the sum of $n_{2}-1$ independent samples of $X, n_{1}$ independent samples of $Z\left(0, \theta_{2}\right)$, and $N-n_{1}-n_{2}-1$ independent samples of $Z\left(\theta_{1}, \theta_{2}\right)$. Since $C(\cdot)$ is convex, we have

$$
C\left(\tau+X^{1}+X^{2}\right)+C\left(\tau+Z^{1}+Z^{2}\right) \geq C\left(\tau+X^{1}+Z^{2}\right)+C\left(\tau+X^{2}+Z^{1}\right) \quad \text { a.s.. }
$$

Taking expectations on both sides yields

$$
\phi\left(n_{2}+1\right)+\phi\left(n_{2}-1\right) \geq \phi\left(n_{2}\right)+\phi\left(n_{2}\right)=2 \phi\left(n_{2}\right),
$$

which is the desired convexity.

The case of $n_{2} \geq N-n_{1}$ is similar, with the following modifications: let $Z^{j}$, for $j=1,2$, be independent samples of $Z\left(0, \theta_{2}\right)$; let $\tau$ be the sum of $n_{2}-1$ independent samples of $X$, and $N-n_{2}-1$ independent samples of $Z\left(0, \theta_{2}\right)$. 
Lemma 7.2 Lemma 4.4 is still valid in the general case when the cost function $C(\cdot)$ is decreasing and convex. Specifically, both (24) and (25) are decreasing in $d_{1}$ and $d_{2}$ and increasing in $n_{2}$.

Proof. For $n_{2} \leq N-n_{1}-1$, let

$$
G_{1}\left(\theta_{1}, \theta_{2}\right):=\phi\left(n_{2}+1, \theta_{1}, \theta_{2}\right)-\phi\left(n_{2}, \theta_{1}, \theta_{2}\right)+r\left(\theta_{1}, \theta_{2}\right)
$$

We want to show that $G_{1}\left(\theta_{1}, \theta_{2}\right)$ is decreasing in $\theta_{1}$ and $\theta_{2}$. Same as before, denote

$$
\eta:=X_{1, n_{2}}+Z_{n_{2}+1, n_{1}+n_{2}}\left(0, \theta_{2}\right)+Z_{n_{1}+n_{2}+2, N}\left(\theta_{1}, \theta_{2}\right)
$$

Note that we have left out the $\left(n_{1}+n_{2}+1\right)^{\text {th }}$ item, for conditioning. Hence,

$$
\begin{array}{rl}
G_{1}\left(\theta_{1}, \theta_{2}\right)=\mathrm{E} & C(\eta+X)-\mathrm{E} C\left(\eta+Z\left(\theta_{1}, \theta_{2}\right)\right)+r\left(\theta_{1}, \theta_{2}\right) \\
=\quad \theta_{1}\left(1-\theta_{2}\right)\left[\mathrm{E} C(\eta+X)-\mathrm{E} C\left(\eta+Y_{1}\right)\right] & \quad+\left(1-\theta_{1}\right) \theta_{2}\left[\mathrm{E} C(\eta+X)-\mathrm{E} C\left(\eta+Y_{2}\right)\right] \\
& +\theta_{1} \theta_{2}\left[\mathrm{E} C(\eta+X)-\mathrm{E} C\left(\eta+Y_{12}\right)\right]+r\left(\theta_{1}, \theta_{2}\right) \\
=\quad c_{i 2} & +\theta_{1}\left(1-\theta_{2}\right)\left[\mathrm{E} C(\eta+X)-\mathrm{E} C\left(\eta+Y_{1}\right)+c_{r 1}\right] \\
& +\theta_{2}\left[\mathrm{E} C(\eta+X)-\mathrm{E} C\left(\eta+Y_{2}\right)+c_{r 2}\right] \\
& +\theta_{1} \theta_{2}\left[\mathrm{E} C\left(\eta+Y_{2}\right)-\mathrm{E} C\left(\eta+Y_{12}\right)+\left(c_{r 12}-c_{r 2}\right)\right] .
\end{array}
$$

Since $C(\cdot)$ is decreasing and convex, comparing the expressions in the first and the third square brackets above with (45) and (47), we know that they are all non-positive. Also, if $\theta_{1}$ is replaced by a larger $\theta_{1}^{\prime}, \eta$ will further decrease, causing the quantities in the two square brackets in question decrease (i.e, becoming more negative). Thus we obtain the desired decreasing property in $\theta_{1}$. For decreasingness in $\theta_{2}$, we can follow the same reasoning, re-organizing the derivation as follows:

$$
\begin{aligned}
G_{1}\left(\theta_{1}, \theta_{2}\right)=c_{i 2} & +\theta_{2}\left(1-\theta_{1}\right)\left[\mathrm{E} C(\eta+X)-\mathrm{E} C\left(\eta+Y_{2}\right)\right. \\
& +\theta_{1}\left[\mathrm{E} C(\eta+X)-\mathrm{E} C\left(\eta+Y_{1}\right)+c_{r 1}\right] \\
& +\theta_{1} \theta_{2}\left[\mathrm{E} C\left(\eta+Y_{1}\right)-\mathrm{E} C\left(\eta+Y_{12}\right)+\left(c_{r 12}-c_{r 1}\right)\right] .
\end{aligned}
$$

Similarly, for $n_{2} \geq N-n_{1}$, let

$$
G_{0}\left(\theta_{1}, \theta_{2}\right):=\phi\left(n_{2}+1, \theta_{1}, \theta_{2}\right)-\phi\left(n_{2}, \theta_{1}, \theta_{2}\right)+r\left(0, \theta_{2}\right)
$$

and denote

$$
\xi:=X_{1, n_{2}}+Z_{n_{2}+2, N}\left(0, \theta_{2}\right) .
$$


Then

$$
\begin{aligned}
G_{0}\left(\theta_{1}, \theta_{2}\right) & =\mathrm{E} C(\xi+X)-\mathrm{E} C\left(\xi+Z\left(0, \theta_{2}\right)\right)+r\left(0, \theta_{2}\right) \\
& =c_{i 2}+\theta_{2}\left[\mathrm{E} C(\xi+X)-\mathrm{E} C\left(\xi+Y_{2}\right)+c_{r 2}\right]
\end{aligned}
$$

is independent of (and hence trivially decreasing in) $\theta_{1}$ and decreasing in $\theta_{2}$.

Note that both $G_{1}$ and $G_{0}$ are increasing in $n_{2}$, following Lemma 7.1. Therefore, following the same argument as in the proof of Lemma 4.4 proves the desired decreasing property in $d_{1}$ and $d_{2}$ and the increasing property in $n_{2}$ of (24) and (25) in the context here.

Here, same as in the earlier case, in order to prove results such as those in Theorem 4.6, the key is to show that $\Psi_{n_{2}}\left(d_{1}, d_{2}\right)-\Phi_{n_{2}}\left(d_{1}, d_{2}\right)$ is decreasing $d_{1}$ and $d_{2}$, and increasing in $n_{2}$. We have already established these properties in Lemma 7.2. Hence, the rest is just to follow the induction steps of Theorem 4.6 (also see the proof of Theorem 3.2).

To summarize, we have

Theorem 7.3 The optimal inspection policy at stage 2 stated in Theorem 4.8 is still optimal under the general cost function, $C(\cdot)$, provided it is decreasing and convex. In particular, Theorem 4.6 still holds under the general cost function. $C(\cdot)$, provided $C(\cdot)$ is a decreasing and convex function.

\section{Concluding Remarks}

Coordination is the key in the quality control of the two-stage system studied here. The optimal policy at the first stage has to take into account the execution at the second stage, in particular, what can and will be optimally carried out. The second stage then continues with what has been done in the first stage; in particular, it gives priority to those units not yet inspected at the first stage. In addition, a threshold structure has been identified with the optimal policies at both stages.

The two properties, $K$-submodularity and convexity, are central to the optimality of the threshold policies at both stages. A heuristic policy, which, for deriving stage-1 policy, treats the stage-2 defect rate as constant, appears to generate near-optimal results. On the other hand, treating the defect rates at both stages as constants simplifies the derivation of the optimal policy, at the price of a more substantial departure from optimality. Both the optimal and the heuristic policies can be precomputed off-line. The computational effort is $O\left(N^{2}\right)$ for stage 1 , and $O\left(N^{4}\right)$ for stage 2 . 


\section{References}

[1] Albin, S.L. And Friedman, D.J., The Impact of Clustered Defect Distributions in IC Fabrication, Management Science, 35 (1989), 1066-1078.

[2] Ballou, D. and Pazer, H., The Impact of Inspector Facility on The Inspection Policy in Serial Production Systems, Management Science, 28 (1982), 387-399.

[3] Bertsekas, D.P., Dynamic Programming and Optimal Control, Athena Scientific, Belmont, MA, 1995.

[4] Blischke, W.R., Mathematical Models for Analysis of Warranty Policies, Math. Comput. Modeling, 13 (1990), 1-16.

[5] Cassandras, C.G., Optimal Policies for the 'Yield Learning' Problem in Manufacturing Systems, IEEE Trans. on Automatic Control, AC-41 (1996), 1210-1213.

[6] Chen, J., Yao, D.D. And Zheng, S., Quality Control for Products Supplied with Warranty. Operations Research, 46 (1998), 107-115.

[7] Eppen, G.D. And Hurst, E.G., Optimal Location of Inspection Stations in A Multistage Production Process, Management Science, 20 (1974), 1194-1200.

[8] Glasserman, P. and Yao, D.D., Monotone Optimal Control of Permutable GSMPs, Mathematics of Operations Research, 19 (1994), 449-476.

[9] Glasserman, P. And Yao, D.D., Subadditivity and Stability of a Class of Discrete Event Systems. IEEE Trans. on Automatic Control, AC-40 (1995), 1514-27.

[10] Lee, H.L., Lot Sizing to Reduce Capacity Utilization in a Production Process with Defective Items, Process Corrections and Rework, Management Science, 38 (1992), 1314-1328.

[11] Lindsay, G.F. And Bishop, A., Allocation of Screening Inspection Effort: A dynamic Programming Approach, Management Science, 10 (1964), 342-352.

[12] Ross, S.M., Stochastic Processes, Wiley, New York, 1983; 2nd edition, 1996.

[13] Ross, S.M., Introduction to Stochastic Dynamic Programming, Academic Press, New York, 1983.

[14] Singpurwalla, N.D. And Wilson, S., The Warranty Problem: Its Statistical and Game Theoretic Aspects, SIAM Review, 35(1) (March, 1993), 17-42.

[15] Topkis, D.M., Minimizing a Submodular Function on a Lattice, Operations Research, 26 (1978), 305-321.

[16] Wald, A., Sequential Analysis, Dover Publications, Inc., 1973.

[17] Walker, D.M.H., Yield Simulation for Integrated Circuits, Kluwer Academic Publishers, Norwell, MA, 1987.

[18] Yao, D.D., And Zheng, S., Sequential Quality Control of a Machining Process. Proceedings of IEEE 34th Conference on Decision and Control, New Orleans, LA, 1995, 3128-33.

[19] Yao, D.D., And Zheng, S., Coordinated Quality Control in a Two-Stage System. Proceedings of IEEE 36th Conference on Decision and Control, San Diego, CA, 1997, 4068-74. 